\title{
Targeting a splicing-mediated drug resistance mechanism in prostate cancer by inhibiting transcriptional regulation by
} PKC $\beta 1$

\author{
James E. Melnyk (iD ${ }^{1}$, Veronica Steri ${ }^{2,3}$, Hao G. Nguyen ${ }^{2,4}$, Y. Christina Hwang (DD $^{2,5}$, John D. Gordan (iD ${ }^{2,5}$, Byron Hann ${ }^{2,3}$,

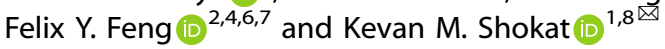

(c) The Author(s) 2022

The androgen receptor (AR) is a central driver of aggressive prostate cancer. After initial treatment with androgen receptor signaling inhibitors (ARSi), reactivation of AR signaling leads to resistance. Alternative splicing of AR mRNA yields the AR-V7 splice variant, which is currently an undruggable mechanism of ARSi resistance: AR-V7 lacks a ligand binding domain, where hormones and anti-androgen antagonists act, but still activates AR signaling. We reveal PKC $\beta$ as a druggable regulator of transcription and splicing at the AR genomic locus. We identify a clinical PKC $\beta$ inhibitor in combination with an FDA-approved anti-androgen as an approach for repressing AR genomic locus expression, including expression of AR-V7, while antagonizing full-length AR. PKC $\beta$ inhibition reduces total AR gene expression, thus reducing AR-V7 protein levels and sensitizing prostate cancer cells to current antiandrogen therapies. We demonstrate that this combination may be a viable therapeutic strategy for AR-V7-positive prostate cancer.

Oncogene (2022) 41:1536-1549; https://doi.org/10.1038/s41388-022-02179-z

\section{INTRODUCTION}

Androgen receptor signaling inhibitors (ARSi) are currently the primary treatment regimen for advanced prostate cancer. These therapies work either by directly antagonizing the AR at its ligandbinding domain (LBD) or by inhibiting androgen synthesis. Such treatments are generally initially successful, but many patients eventually relapse and develop lethal, metastatic castrationresistant prostate cancer (CRPC), which thrives even in a reduced-hormone environment [1]. CRPC progresses through several possible mechanisms, including complete AR independence, LBD mutations that relax steroid-binding specificity, adrenal or intra-tumoral androgen synthesis, amplification of the AR gene body and its enhancers, and the AR-V7 alternative splice variant [1-4].

The presence of the AR-V7 splice variant is associated with resistance to ARSi therapies and clinically poor outcomes [3]. ARV7 is a constitutively active, androgen-independent transcription factor that lacks its LBD but retains its DNA-binding domain and is thus able to circumvent the actions of current anti-androgen therapies that target the LBD [5-7]. Elegant prior work reveals that AR expression increases during androgen blockade and concomitantly leads to AR alternative splicing and production of AR-V7 [59]. While anti-androgen therapies block activation of full-length AR, AR-V7 which is also produced lacks the LBD resulting in an undruggable isoform of the druggable $A R$ oncogene $[5,7]$, and has established an unmet need for novel therapeutic approaches to target AR-V7.

Targeting AR-V7 is currently an active and dynamic area of drug discovery. Drugs with several conceptually distinct approaches have been identified and pursued: (1) small molecules that bind to the AR-V7 protein despite its lack of known small-moleculetargetable features [10-13], (2) small molecules which lead to degradation of AR-V7 [14-16], (3) agents that act indirectly to target AR-V7 [17, 18], and (4) antisense oligonucleotides and small molecules that modulate AR pre-mRNA alternative splicing [1922]. To date, efforts to target AR pre-mRNA in prostate cancer have been dominated by antisense oligonucleotide strategies that appear promising but have yielded very limited clinical success [19-21, 23, 24]. We were therefore encouraged to evaluate a small molecule approach to target transcription and splicing of AR premRNA in AR-V7-positive prostate cancer.

The AR has a well-established role as an androgen-dependent transcriptional activator, and the mechanistic details for the activation of its targets are well studied [25-29]. But importantly, the $A R$ is also responsible for androgen-dependent transcriptional repression. In its transcriptional suppressor role, the androgenbound full-length AR recruits chromatin-modifying complexes to genomic targets to remove activating histone marks and recruits transcription suppressors $[29,30]$. Genes repressed by the AR notably include the AR itself; in a high-androgen environment, the

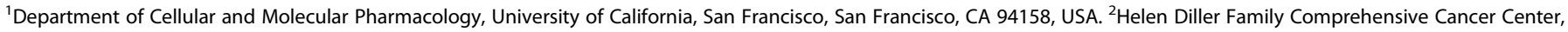

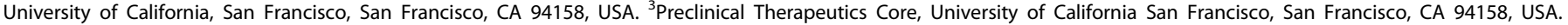

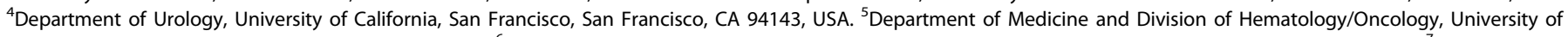

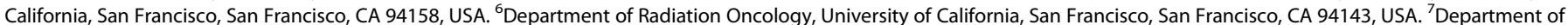

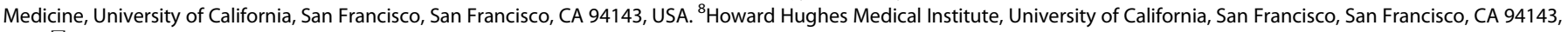
USA. ${ }^{\circledR}$ email: Kevan.shokat@ucsf.edu
} 
A

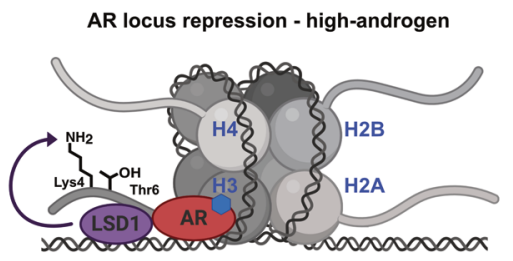

AR locus activation - low-androgen

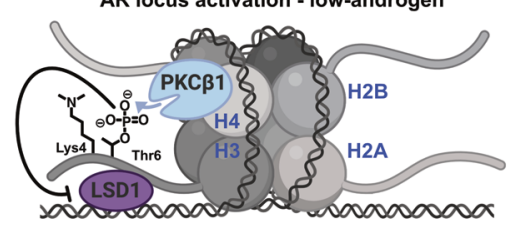

AR locus activation inhibited by PKC $\beta$ i

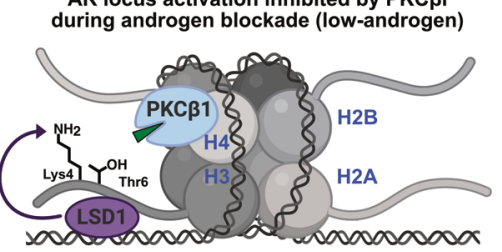

AR locus transcription ARE gene

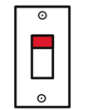

OFF

AR Iocu AR locus
transcription

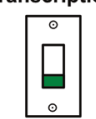

ON transcription

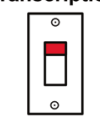

OFF ARscription

D
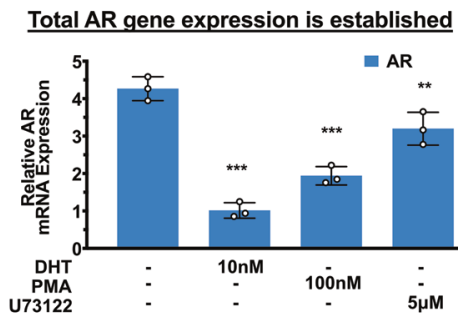

- $\mathrm{AR}$

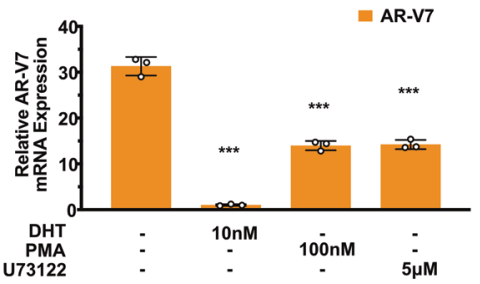

B

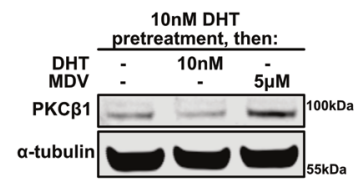

C

Total AR gene expression is established

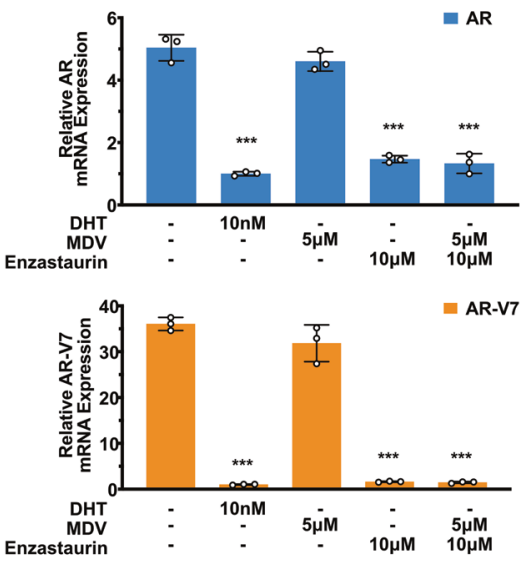

E

10nM DHT pretreatment, then treated as indicated: 100 nM PMA time course
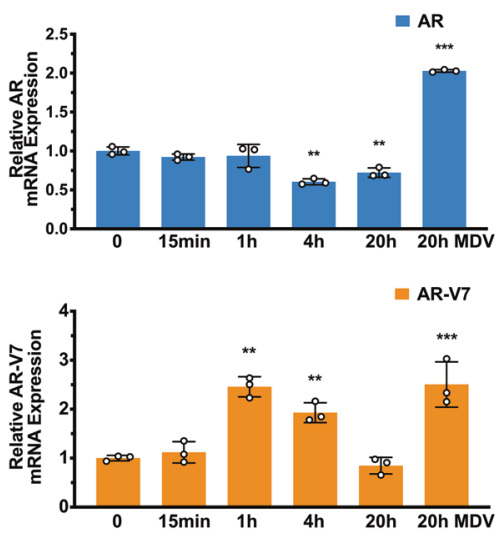

$\mathbf{F}$

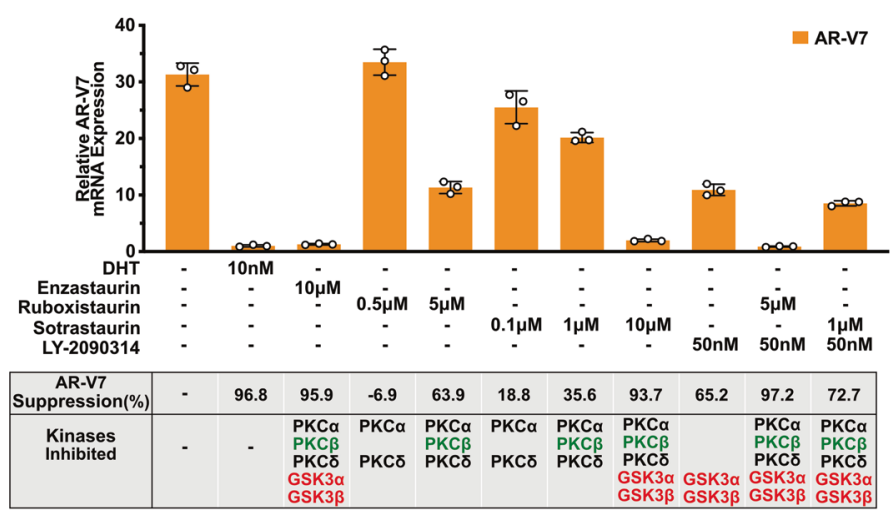

androgen-bound full-length $A R$ inhibits transcription at the $A R$ genomic locus by binding repressive sites along intron 2 , causing a reduction in total AR mRNA transcripts in a negative feedback loop [6, 31]. Conversely, anti-androgen therapies such as enzalutamide (MDV) block androgen-binding and localization to the AR genomic locus, resulting in increased transcriptional activity at the $A R$ locus and an increase in total AR mRNA transcripts, including those of the AR-V7 splice variant that drives resistance to the same therapies. We hypothesized that druggable kinases may regulate transcription and splicing at the AR genomic 
Fig. 1 PKC $\beta 1$ inhibition reduces expression of the AR genomic locus and decreases AR and AR-V7 mRNA transcripts. A Images of a nucleosome with each of the four core histone proteins indicated. Established mechanism of AR locus repression in high-androgen conditions (top). Proposed mechanism of AR locus activation by PKC $\beta 1$ in low-androgen conditions (middle). Proposed inhibition of AR locus activation during androgen blockade by a PKC $\beta 1$ inhibitor (bottom). The blue hexagon represents DHT and the green triangle represents a PKC $\beta 1$ inhibitor. B VCaP cells cultured in RPMI 1640 supplemented with $5 \%$ CSS for $48 \mathrm{~h}$ were treated for $24 \mathrm{~h}$ with DHT, washed out, then treated as indicated for an additional $24 \mathrm{~h}$ and immunoblotted for PKC 31 . C, D VCaP cells cultured in RPMI 1640 supplemented with $5 \%$ CSS for $48 \mathrm{~h}$ were treated in three biological replicates for $24 \mathrm{~h}$ as indicated and analyzed by RT-qPCR for AR and AR-V7 mRNA transcript levels. Data are mean \pm SD. P-values are relative to vehicle ${ }^{* * *} p$-value $\left.<0.0001\right)$. All mRNA expression levels are relative to GAPDH and normalized to the response for DHT. E VCaP cells cultured in RPMI 1640 supplemented with $5 \%$ CSS for $48 \mathrm{~h}$, pre-treated with $10 \mathrm{nM}$ DHT for $24 \mathrm{~h}$, and then washed out and treated with $100 \mathrm{nM}$ PMA for the indicated timepoints, or with $5 \mu \mathrm{M}$ MDV for $20 \mathrm{~h}$ and analyzed by RT-qPCR for AR and AR-V7 mRNA transcript levels. Data are mean \pm SD. P-values are relative to time $0\left({ }^{* *} p\right.$-value $<0.005 ;{ }^{* *} p$-value $\left.<0.0001\right)$. All mRNA expression levels are relative to GAPDH and normalized to the response for time 0 . F VCaP cells cultured in RPMI 1640 supplemented with $5 \%$ CSS for $48 \mathrm{~h}$ were treated in three biological replicates for $24 \mathrm{~h}$ with PKC and GSK3 inhibitors and analyzed by RT-qPCR for AR-V7 mRNA transcript levels. Table shows \% suppression of AR-V7 mRNA transcripts relative to vehicle. Data are mean \pm SD. All mRNA expression levels are relative to GAPDH and normalized to the response for DHT.

locus to mediate the changes in AR gene expression observed in CRPC.

On the basis of this hypothesis, we evaluated potential kinase targets and selected the protein kinase PKC $\beta 1$ for investigation. PKC $\beta 1$ is reported to be associated with active transcription in prostate cancer and phosphorylates histone H3T6 [27]. H3T6 phosphorylation blocks lysine demethylases from removing mono- and dimethyl marks at $\mathrm{H} 3 \mathrm{~K} 4$, preventing transcriptional repression [27]. We, therefore, hypothesized that PKC $\beta 1$ could be present at the AR genomic locus during androgen blockade and promoting transcription by phosphorylating H3T6. In this study, we investigate the use of PKC $\beta$ inhibition to reduce antiandrogen-driven transcriptional activation at the AR genomic locus. We find that inhibiting PKC $\beta$ reduces total $A R$ transcript levels, including AR-V7 splice variant levels, and sensitizes AR-V7positive prostate cancer cells to existing anti-androgen therapies.

\section{RESULTS \\ Androgen negatively regulates AR gene expression in VCaP cells}

The VCaP prostate cancer cell line is a metastatic CRPC model that expresses full-length wild-type (WT) $A R$ and the alternatively spliced AR-V7 isoform [5, 7-9]. This cell line has an amplification of the AR locus and is androgen-responsive [32]. When VCaP cells are cultured in growth medium supplemented with charcoal-stripped serum (CSS), a low-androgen environment, full-length AR and ARV7 mRNA and protein levels are elevated [5, 7-9]. Conversely, after treatment for $24 \mathrm{~h}$ with the AR agonist dihydrotestosterone (DHT), full-length $A R$ and AR-V7 mRNA and protein levels are markedly reduced in a dose-dependent manner (Supplementary Fig. S1A and S1B; Supplementary Table S1).

\section{PKC $\beta 1$ expression increases during androgen blockade}

Prior work revealed that prolonged exposure to high-androgen environments leads to binding of full-length AR at repressive sites along intron 2 of the AR genomic locus, which recruits the lysine demethylase LSD1 (KDM1A) to remove methyl marks from histone $\mathrm{H} 3 \mathrm{~K} 4$ as represented in Fig. 1A (top), suppressing AR gene expression [6]. The protein kinase PKC $\beta 1$ is reported to promote active transcription by phosphorylating histone H3T6, which blocks LSD1 demethylase activity at H3K4 [27]. We, therefore, hypothesized that PKC $\beta 1$ is present at the AR genomic locus during low-androgen conditions, promoting transcription and increasing total AR transcript levels by phosphorylating histone H3T6 (Fig. 1A (middle)). We assessed PKC $\beta 1$ protein expression in the VCaP cell line and found that it is downregulated by DHT and upregulated by MDV (Fig. 1B). This observation aligns with a prior report that MDV induces PKC-family members in prostate cancer cells [33]. We propose PKC $\beta 1$ as an important component of the low-androgen stress response that upregulates AR gene expression and increases full-length AR and AR-V7 protein levels during AR antagonism.

\section{Enzastaurin reduces AR and AR-V7 mRNA transcript levels}

We hypothesized that inhibition of PKC 31 -mediated H3T6 phosphorylation would allow demethylation of H3K4, thus decreasing transcriptional activity at the AR genomic locus (Fig. $1 \mathrm{~A}$ (bottom)), and consequently decreasing both AR and AR-V7 mRNA transcripts. We assessed the ability of the PKC $\beta$ inhibitor enzastaurin to suppress both full-length AR and AR-V7 mRNA transcripts. VCaP cells were cultured for $48 \mathrm{~h}$ in a low-androgen environment to maximize the low-androgen stress response that increases transcription at the AR genomic locus [5, 7-9]. Cells were subsequently treated with either MDV, enzastaurin, or both (Fig. 1C). This assay is designed to mimic a clinical situation in which prostate cancer is highly expressing full-length $A R$ and AR-V7. As hypothesized, enzastaurin alone or in combination with MDV reduced the level of both full-length $A R$ and AR-V7 mRNA transcripts. Full-length AR transcripts decreased by 3.4-fold and 3.9-fold respectively relative to the vehicle, while AR-V7 mRNA transcripts decreased more starkly by 22.2 -fold and 24.5 -fold respectively.

We subsequently assessed the ability of enzastaurin to suppress full-length $A R$ and AR-V7 mRNA transcripts while the lowandrogen stress response is building, and therefore transcription at the AR genomic locus is increasing. In this assay, VCaP cells were pre-treated with DHT to suppress total AR mRNA transcripts. DHT was subsequently washed out, and the cells were treated with either additional DHT, MDV, enzastaurin, or enzastaurin in combination with MDV. In these conditions, androgen blockade by MDV alleviates androgen-dependent AR genomic locus repression causing an increase in transcriptional activity at the AR genomic locus $[5,6]$. This assay is designed to resemble a clinical situation in which the AR-V7 splice variant is emerging during anti-androgen therapy. Enzastaurin was able to reduce MDV-induced expression of full-length AR mRNA transcripts by 1.9-fold and AR-V7 mRNA transcripts by 4.9-fold, relative to MDV (Supplementary Fig. S2A). Collectively, the data demonstrate that enzastaurin reduces total AR mRNA transcripts, but with a greater magnitude effect for AR-V7.

\section{Enzastaurin activity is mediated through PKC $\beta$ inhibition}

Enzastaurin was developed as a clinical PKC $\beta$ inhibitor, however, it also exhibits potent inhibition of PKCa, PKC $\delta$, GSK3a, and GSK3 $\beta$ [34]. First, to test whether the effects of enzastaurin on $A R$ transcription are driven by PKC-family kinases, we assessed AR gene expression after chemical knockdown of PKC-family kinases with phorbol 12-myristate 13-acetate (PMA), or after treatment with the phospholipase $C$ inhibitor, U73122, to respectively degrade PKC-family kinases or to suppress second messenger synthesis that activates PKC-family kinases. PMA activates both 
A

AR locus - region of intron 2

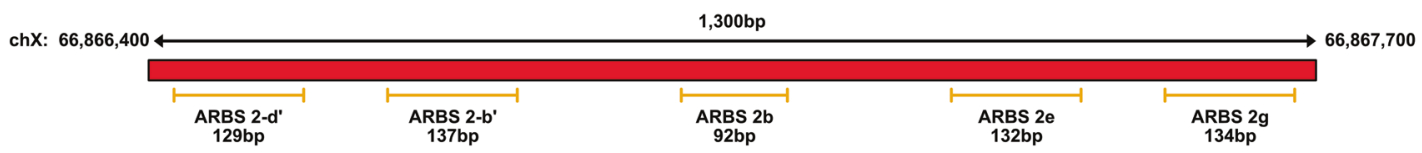

B

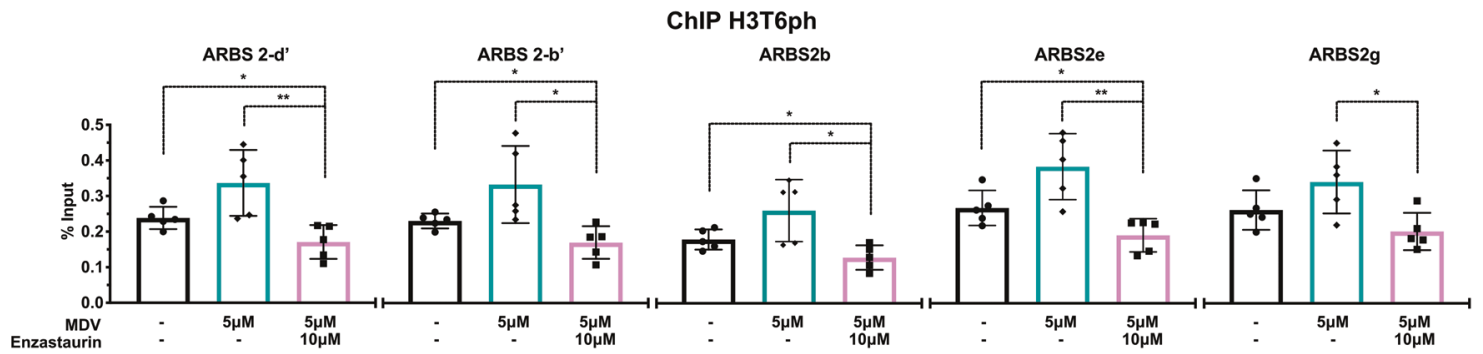

ChIP H3K4Me2

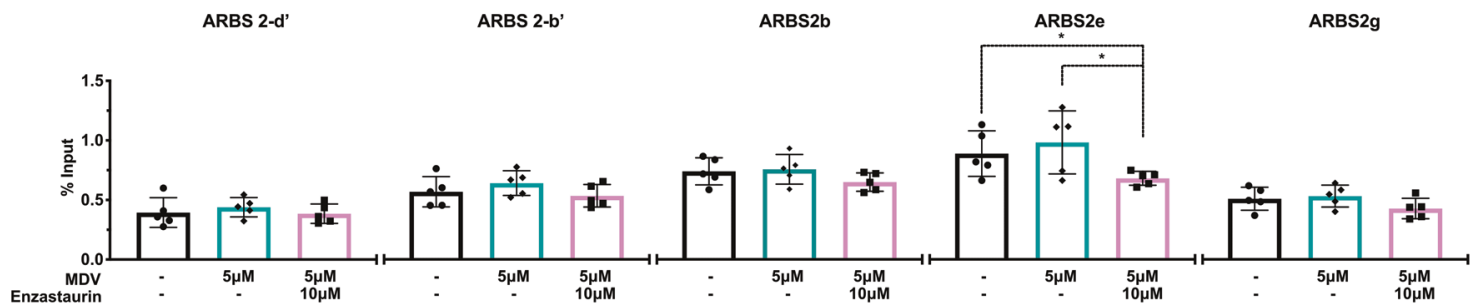

ChIP H3K4Me1

ARBS 2-d' ARBS 2-b' ARBS2b

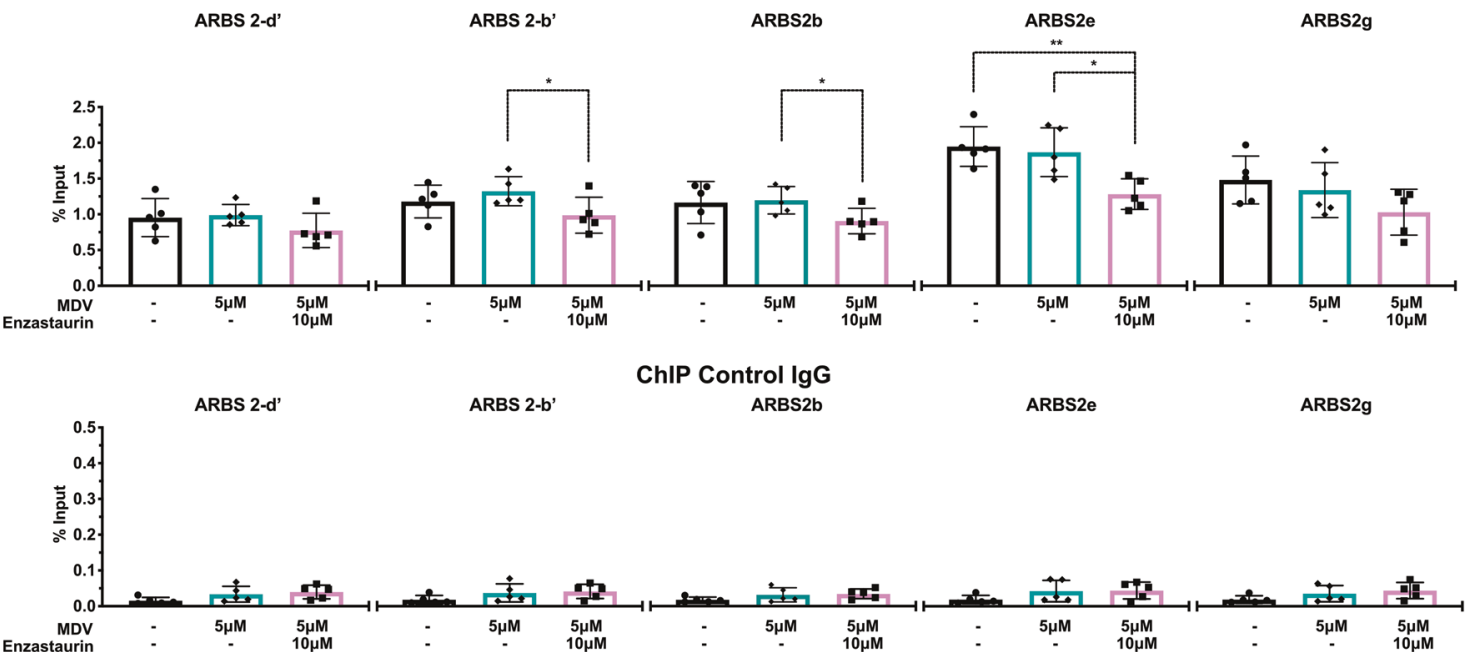

Fig. 2 PKC 1 inhibition reduces H3T6 phosphorylation and H3K4 methylation at the AR genomic locus. A Intron 2 at the AR genomic locus with primers spanning the indicated region for ChIP-qPCR analysis (Assembly GRCh37.p13). B VCaP cells cultured in RPMI1640 supplemented with $5 \%$ CSS for $48 \mathrm{~h}$ and then treated as indicated for $24 \mathrm{~h}$. Samples $(N=5$ biological replicates) processed according to the Zymo-Spin ChIP Kit with H3T6ph, H3K4Me2, H3K4Me1, and rabbit IgG antibodies. The antibody precipitated chromatin was de-crosslinked, purified, and analyzed by qRT-PCR using the primers against the regions indicated. Data is reported as percent of input and are mean \pm SD $\left({ }^{*} p\right.$-value $<0.05,{ }^{* *} p$-value $\left.<0.01\right)$.

conventional and novel PKC-family kinases, which leads to their rapid degradation (Supplementary Fig. S2B) [35-38]. These kinases have cellular half-lives on the order of days in tissue culture conditions, making genetic knockdown difficult since PKC-family protein levels persist even with successful gene silencing. The use of PMA, therefore, circumvents difficulties with genetic knockdown [37]. U73122 is an inhibitor of Phospholipase C, which hydrolyzes phosphatidylinositol 4,5-bisphosphate (PIP2) to produce diacylglycerol (DAG) and inositol 1,4,5-trisphosphate (IP3), which stimulates the release of $\mathrm{Ca}^{2+}$. Both DAG and $\mathrm{Ca}^{2+}$ are second messengers for conventional and novel PKC family member activation [39]. Treatment with either PMA or U73122 decreased AR-V7 mRNA transcript levels (Fig. 1D). Due to the initial, robust activation of PKC family members induced by PMA prior to degradation, we also performed a time-course experiment to determine if PMA treatment initially increases AR-V7 mRNA transcripts at shorter time points. Our results reveal an increase in AR-V7 mRNA transcript levels after one to four hours. Importantly, in this assay the PMA-induced increase is similar to that observed for MDV. However, an increase in full-length AR mRNA levels is not observed at shorter PMA treatment time points, and a reduction in full-length AR mRNA levels is observed after four hours (Fig. 1E). 
A Total AR gene expression is established Steriod-depleted medium

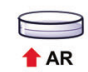$$
\text { AR-V7 }
$$$$
48 \mathrm{~h}
$$
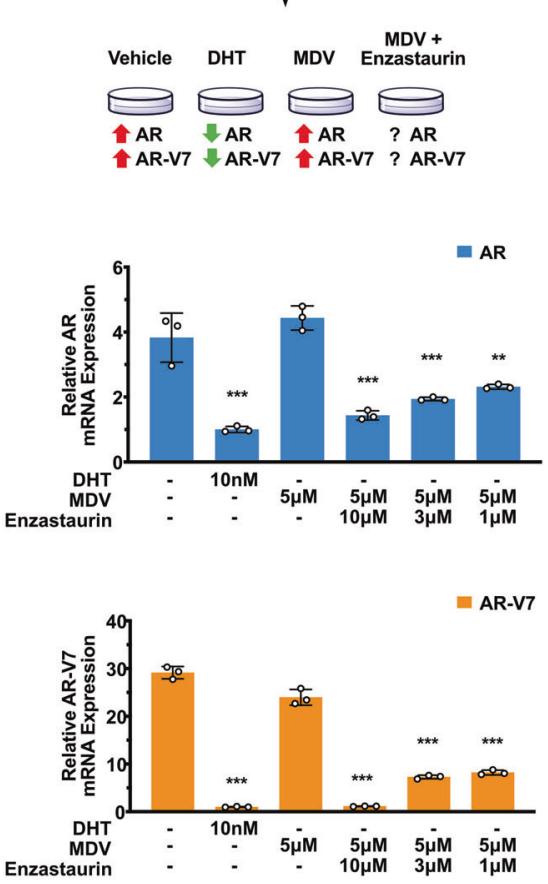

C Total AR gene expression is established

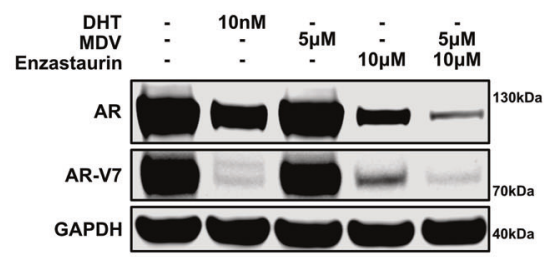

E

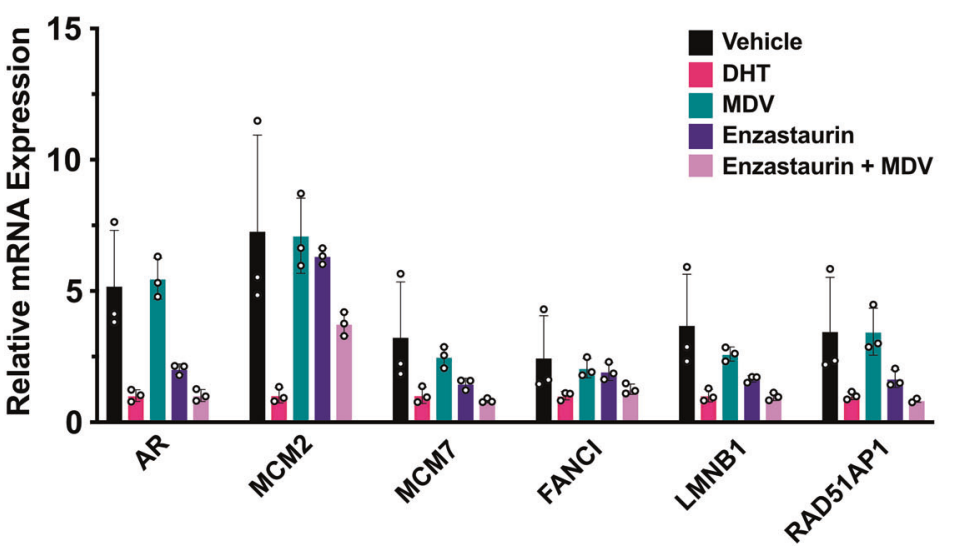

We then evaluated the dose-dependent effects of ruboxistaurin and sotrastaurin, PKC-family inhibitors with differing affinities for PKCa, PKC $\beta$, and PKC $[34]$, on AR-V7 mRNA transcript levels. We found that inhibitor doses at which PKC $\beta$ should be inhibited reduced AR-V7 mRNA levels, while PKCa and PKC $\delta$ inhibition

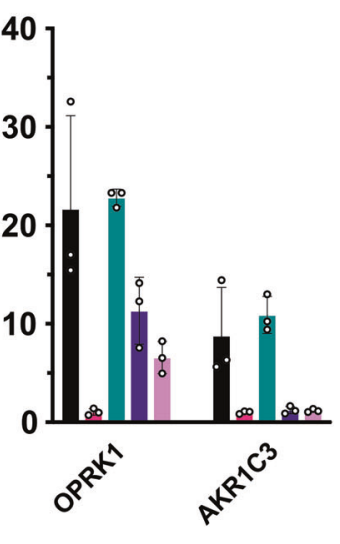

Total AR gene expression is building

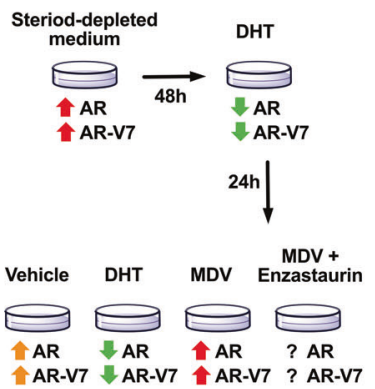

10nM DHT pretreatment, then treated as indicated:

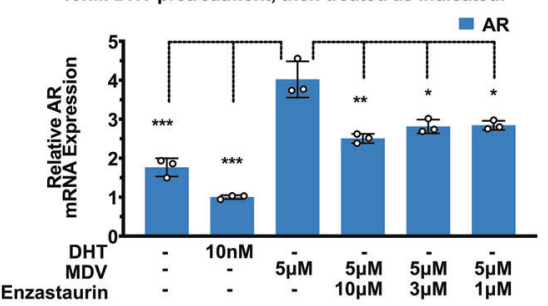

10nM DHT pretreatment, then treated as indicated:

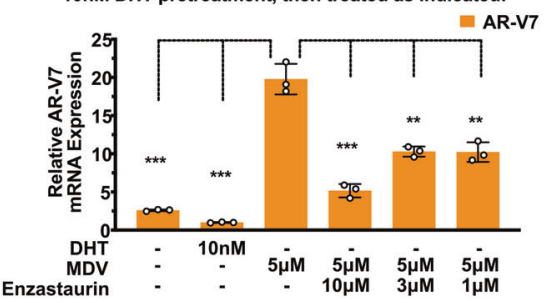

D

Total AR gene expression is building

10nM DHT pretreatment, then treated as indicated:

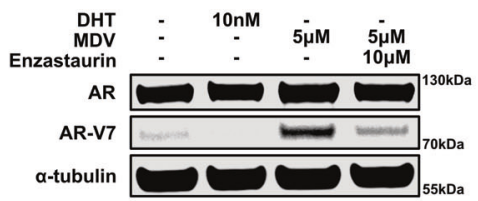

alone had little effect (Fig. 1F). Additionally, we analyzed LY2090314 a potent GSK3 $\alpha$ and GSK3 $\beta$ inhibitor, and found GSK3a and GSK3 $\beta$ inhibition also yields a partial reduction in AR-V7 mRNA transcript levels. However, the most potent reductions of AR-V7 mRNA levels are observed in conditions when PKC $\beta, G S K 3 a$, 
Fig. 3 Enzastaurin effectively suppresses AR and AR-V7 mRNA and protein levels, and decreases mRNA transcripts of other androgendependent AR suppressed targets that are de-repressed during anti-androgen therapy. A VCaP cells cultured in RPMI1640 supplemented with $5 \%$ CSS for $48 \mathrm{~h}$ were treated in three biological replicates for $24 \mathrm{~h}$ as indicated and analyzed by RT-qPCR for AR and AR-V7 mRNA transcript levels. Data are mean \pm SD. P-values are relative to vehicle $\left({ }^{*} p\right.$-value $<0.05 ;{ }^{* *} p$-value $<0.005 ;{ }^{* * *} p$-value $\left.<0.0001\right)$. All mRNA expression levels are relative to GAPDH and normalized to the response for DHT. B VCaP cells cultured in RPMI 1640 supplemented with $5 \%$ CSS for $48 \mathrm{~h}$, pre-treated with DHT for $24 \mathrm{~h}$, and then washed out and treated as indicated in three biological replicates for an additional $24 \mathrm{~h}$ and analyzed by RT-qPCR for AR and AR-V7 mRNA transcript levels. Data are mean \pm SD. P-values are relative to MDV $\left({ }^{*} p\right.$-value $<0.05 ;{ }^{* *} p$ value $<0.005 ;{ }^{* * *} p$-value $\left.<0.0001\right)$. All mRNA expression levels are relative to GAPDH and normalized to the response for DHT. C VCaP cells cultured in RPMI1640 supplemented with $5 \%$ CSS for $48 \mathrm{~h}$ were treated as indicated for $72 \mathrm{~h}$ and then immunoblotted for AR and AR-V7. D VCaP cells cultured in RPMI 1640 supplemented with $5 \%$ CSS for $48 \mathrm{~h}$, pre-treated with DHT for $24 \mathrm{~h}$, washed out, treated as indicated for an additional $24 \mathrm{~h}$, and immunoblotted for AR and AR-V7. E VCaP cells cultured in RPMI 1640 supplemented with $5 \%$ CSS for $48 \mathrm{~h}$ were treated with vehicle, $10 \mathrm{nM}$ DHT, $5 \mu \mathrm{M}$ MDV, $10 \mu \mathrm{M}$ Enzastaurin, or $5 \mu \mathrm{M}$ MDV $+10 \mu \mathrm{M}$ Enzastaurin in three biological replicates for $24 \mathrm{~h}$ and then analyzed by RT-qPCR for mRNA transcript levels of DHT-AR repressed genes. Data are mean \pm SD. All mRNA expression levels are relative to GAPDH and normalized to the response for DHT.

and GSK3 $\beta$ are all simultaneously inhibited $(10 \mu \mathrm{M}$ enzastaurin, $10 \mu \mathrm{M}$ sotrastaurin, $5 \mu \mathrm{M}$ ruboxistaurin $+50 \mathrm{nM}$ LY-2090314, and $1 \mu \mathrm{M}$ sotrastaurin $+50 \mathrm{nM}$ LY-2090314) (Fig. 1F). We then used a multiplexed inhibitor bead (MIB) column strategy [40] to compare kinase activity profiles during MDV or DHT treatment. We found that GSK3a, GSK3 $\beta$, and several $\mathrm{Ca}^{2+}$-stimulated kinases are activated during androgen blockade. We were not able to detect PKC $\beta 1$ in this assay (Supplementary Fig. S2C), although this may be due to a low expression level and the presence of other PKCfamily members that make detection difficult. In summary, we conclude that the effect of enzastaurin on the AR is primarily mediated by PKC $\beta$ inhibition, but is enhanced by its polypharmacology against GSK3a and GSK3 $\beta$.

PKC $\beta$ inhibition reduces histone H3T6 phosphorylation and decreases histone H3K4 methylation at the AR genomic locus Next, we wished to evaluate the mechanism of AR genomic locus regulation by PKC $\beta$. We utilized a ChIP-qPCR assay with primers spaced along intron 2 of the AR genomic locus in the region previously reported to contain AR regulatory elements (Fig. 2A) [6]. Our assay reveals that enzastaurin and MDV in combination decreases histone H3T6 phosphorylation across all primer sets in this region relative to MDV alone. Further, the combination of enzastaurin and MDV reduces both histone H3K4 di- and monomethylation at select primer sets relative to MDV alone. These observations are consistent with our proposed mechanism, where PKC $\beta$ inhibition decreases histone H3T6 phosphorylation resulting in an increase in LSD1 activity and a concomitant reduction in histone H3K4 methylation. These trends are not observed for the control IgG (Fig. 2B). Finally, we evaluated LSD1 at the AR genomic locus in our assay, and observed the presence of LSD1 in all treatment conditions and across all primer sets. Further, we generally observed a slight enrichment of LSD1 in the enzastaurin + MDV combination, and for MDV alone, when compared to vehicle. These trends were not observed for the control IgG (Supplementary Fig. S2D).

Enzastaurin exhibits dose-dependent inhibition of AR and ARV7 expression and suppresses rebound expression of ARrepressed genes

Having established that enzastaurin represses expression of fulllength $A R$ and $A R-V 7$, we next investigated the effect in more detail. We assessed the dose-dependent effects of enzastaurin in combination with MDV on full-length $A R$ and AR-V7 mRNA transcript levels both when the low-androgen stress response is established, and when it is building, and observed a dose dependency for both conditions. Even at our lowest assay concentration of enzastaurin $(1 \mu \mathrm{M})$ in combination with MDV, full-length AR mRNA transcript levels were reduced 1.7-fold and AR-V7 mRNA transcript levels were reduced 3.6-fold relative to vehicle when the low-androgen stress response is established (Fig. $3 \mathrm{~A})$, and full-length AR mRNA transcript levels were reduced 1.4- fold and AR-V7 mRNA transcript levels were reduced 1.9-fold relative to MDV when the low-androgen stress response is building (Fig. 3B). Further, in each condition the magnitude of the effect was greatest for AR-V7. Next, we evaluated if a reduction in AR and AR-V7 mRNA transcripts is associated with a decrease in $A R$ and $A R-V 7$ protein levels. We were able to observe a very clear decrease in AR and AR-V7 protein levels in the presence of our combinations (Figs. 3C, D). Enzastaurin also yielded a dosedependent reduction in AR-V7 protein levels in the combination treatments (Supplementary Fig. S3A). Further, when cells are treated with enzastaurin alone, a decrease in AR-V7 protein levels relative to MDV alone is observed, demonstrating that this effect is fully dependent on the PKC $\beta$ inhibitor (Fig. 3C; Supplementary Fig. S3B).

Next, we evaluated our combination in another AR-V7 positive cell line that is resistant to anti-androgen treatment. The 22RV1 prostate cancer cell line expresses full-length AR and AR-V7 splice variant and includes a drug-binding-resistant somatic mutation in the full-length AR LBD (H875Y). The cell line also contains a $35 \mathrm{~kb}$ intragenic tandem duplication of the $A R$ gene that results in deregulation of $A R$ splicing and contributes to $A R$ alternative splicing and AR-V7 mRNA transcripts. This duplication event encompasses exon 3 and the neighboring sequences, including cryptic exon 3, which is found in AR-V7 [41, 42]. In 22RV1 cells, enzastaurin alone and in combination with MDV significantly decreased AR-V7 mRNA levels but not full-length AR mRNA levels, relative to vehicle and MDV. (Supplementary Fig. S3C). A reduction in AR-V7 protein levels was also observed (Supplementary Fig. $\mathrm{S} 3 \mathrm{D})$. We suspect the ability of enzastaurin to reduce AR-V7 in the 22RV1 cell line indicates that despite AR genomic rearrangements, transcriptional regulatory elements at the AR locus-including the role of $\mathrm{PKC} \beta 1$-remain intact.

We hypothesized that the mechanism of AR genomic locus repression (Fig. 1A) may also be reflected at other genomic loci that are known targets of androgen-dependent repression by the full-length AR [29]. We, therefore, tested enzastaurin alone and in combination with MDV against a subset of these targets, and in almost all cases observed a reduction in mRNA transcript levels when compared to vehicle or MDV (Fig. 3E). Interestingly, targets suppressed by our combination include proteins involved in genome replication ( $\mathrm{MCM} 2$ and $\mathrm{MCM} 7$ ), in DNA damage response, and the repair of double-stranded breaks (FANCI and RAD51AP1), and metabolic enzymes involved in androgen synthesis (AKR1C3). The data indicates that combining enzastaurin with MDV can also mitigate the increases in transcription observed in response to MDV at other AR-repressed genomic loci. Finally, we evaluated expression of the AR target genes KLK2, KLK3, TMPRSS2, and ZBTB16, which demonstrate androgen-dependent activation (Supplementary Fig. S3E). Further, androgen-dependent AR activation of KLK2, KLK3, and TMPRSS2 is enhanced by $P K C \beta 1$ activity [27]. ZBTB16 activation has not been evaluated in this context. We observed that MDV inhibited transcription of all four 
A
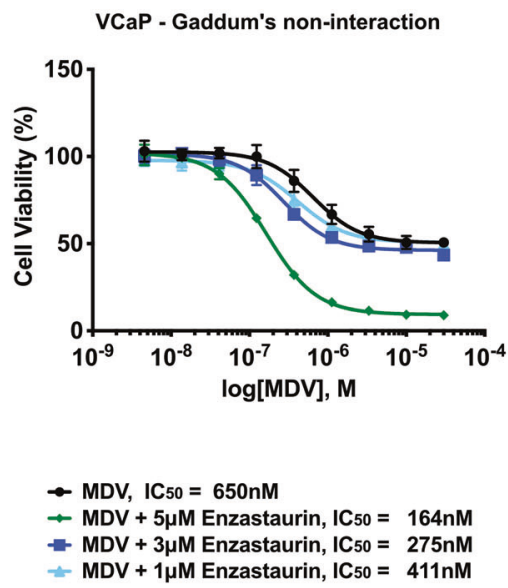

B

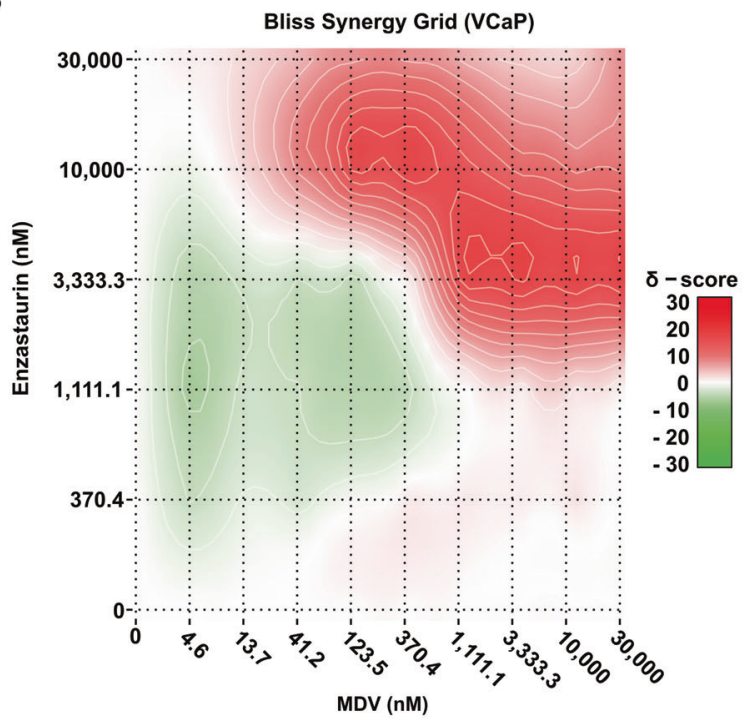

C

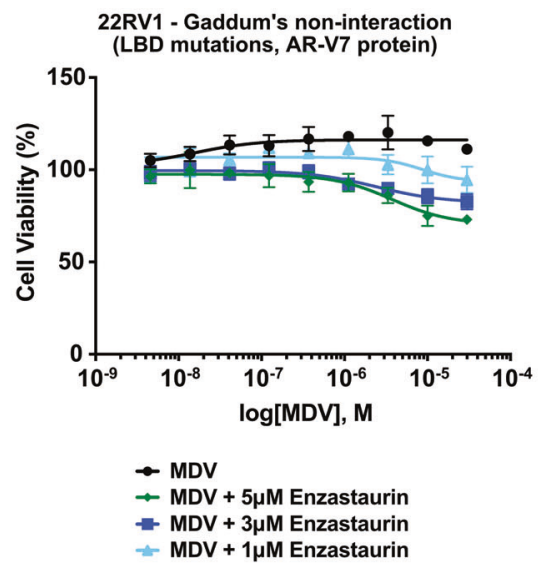

D

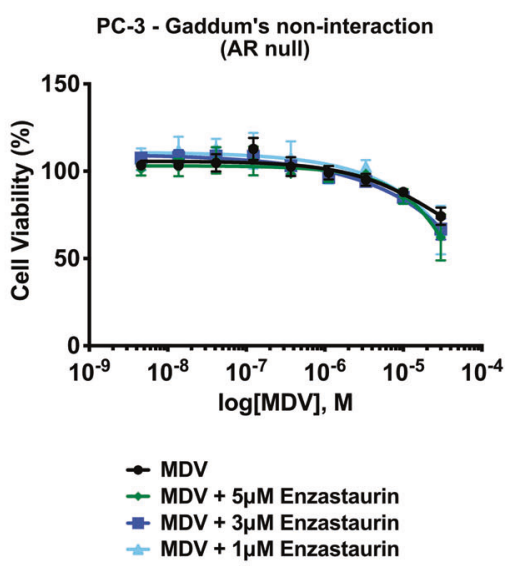

E

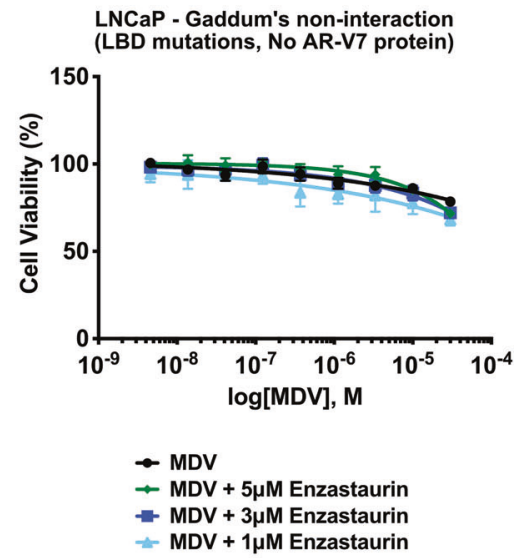

Fig. 4 Enzastaurin and MDV combinations demonstrate synergy in the AR-V7 expressing VCaP cell line but not in other prostate cancer cell lines. A VCaP (5000 cells/well) cells were cultured in RPMI 1640 supplemented with $5 \%$ CSS for $48 \mathrm{~h}$ in 96 -well plates. In the presence of

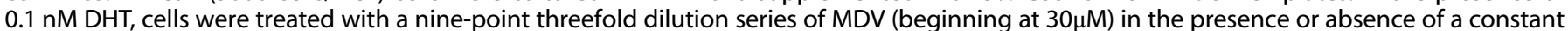
concentration of Enzastaurin for three days. Cell viability was subsequently measured in a CellTiter-Glo bioluminescence assay. Data are mean \pm SD $(N=3$ biological replicates). B VCaP ( 5000 cells/well) cells were cultured in RPMI1640 supplemented with $5 \%$ CSS for $48 \mathrm{~h}$ in 96 well plates. In the presence of $0.1 \mathrm{nM} \mathrm{DHT}$, cells were treated in combination with MDV and Enzastaurin (beginning at $30 \mu \mathrm{M}$ ) in a threefold dilution series checkerboard assay for five days. Cell viability was subsequently measured in a CellTiter-Glo bioluminescence assay $(N=3$ biological replicates) and Bliss synergy scores were calculated. C 22RV1 cells express AR-V7 protein and contain LBD mutation H875Y. 22RV1 (2000 cells/ well) cells were cultured in RPMI 1640 supplemented with $5 \%$ CSS for $48 \mathrm{~h}$ in 96 well plates. In the presence of $0.1 \mathrm{nM}$ DHT, cells were treated with a nine-point threefold dilution series of MDV (beginning at $30 \mu \mathrm{M}$ ) in the presence or absence of a constant concentration of Enzastaurin for five days. Cell viability was subsequently measured in a CellTiter-Glo bioluminescence assay. Data are mean \pm SD $(N=3$ biological replicates). D PC-3 cells are AR null. PC3 (1000 cells/well) were cultured in RPMI1640 supplemented with $5 \%$ CSS for $48 \mathrm{~h}$ in 96 well plates. In the presence of $0.1 \mathrm{nM}$ DHT, cells were treated with a nine-point threefold dilution series of MDV (beginning at $30 \mu \mathrm{M}$ ) in the presence or absence of a constant concentration of Enzastaurin for three days. Cell viability was subsequently measured in a CellTiter-Glo bioluminescence assay. Data are mean \pm SD ( $N=3$ biological replicates). E LNCaP cells do not have AR-V7 protein and contain LBD mutation T878A. LNCaP (5000 cells/well) cells were cultured in RPMI1640 supplemented with $5 \%$ CSS for $48 \mathrm{~h}$ in 96 well plates. In the presence of $0.1 \mathrm{nM}$ DHT, cells were treated with a nine-point threefold dilution series of MDV (beginning at $30 \mu \mathrm{M}$ ) in the presence or absence of a constant concentration of Enzastaurin for three days. Cell viability was subsequently measured in a CellTiter-Glo bioluminescence assay. Data are mean \pm SD $(N=3$ biological replicates).

genes, and that this inhibitory effect was further enhanced by enzastaurin.

\section{MDV and enzastaurin combinations demonstrate synergy in AR-V7 positive prostate cancer cells}

To test the hypothesis that reducing full-length $A R$ and AR-V7 splice variants during androgen blockade will increase antiandrogen effectiveness in our VCaP cell line, we assessed our combinations in several drug synergy assays. First, we assessed synergy by Gaddum's non-interaction, also known as the Highest Single Agent model. In this model, a synergistic combination will yield a greater effect than a single agent alone at the same concentrations $[43,44]$. We, therefore, determined the $I C_{50}$ values on VCaP cell viability for serial dilutions of MDV alone and at constant concentrations of enzastaurin. As expected, enzastaurin improved the effectiveness of MDV when measured by $I_{50}$ (Fig. $4 A)$. Second, we assessed the synergy of our combination in a checkerboard assay using the SynergyFinder web application and 
A<smiles>Cc1ncsc1-c1ccc(CNC(=O)[C@@H]2CC(O)CN2C(=O)[C@@H](NC(=O)COCCCOCCCCCOc2ccc(N3C(=S)N(c4ccc(C#N)c(C(F)(F)F)c4)C(=O)C3(C)C)cc2)C(C)(C)C)cc1</smiles>

B

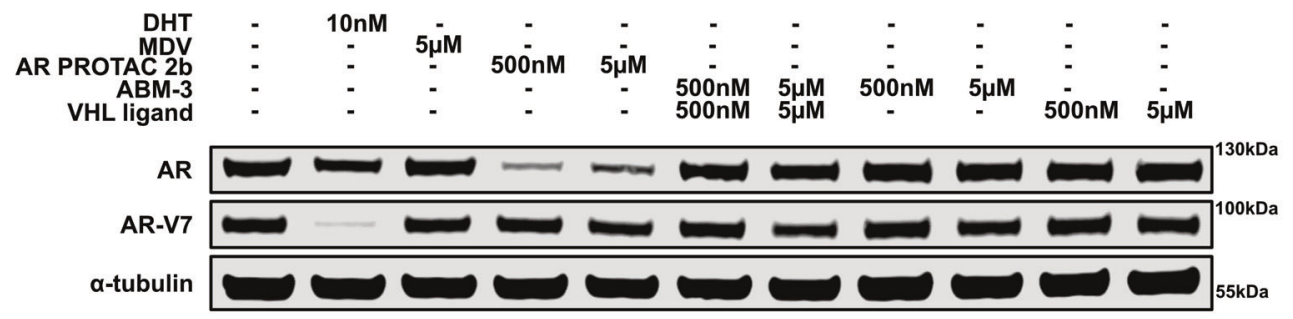

\section{C}

Total AR gene expression is building 10nM DHT pretreatment, then treated as indicated:

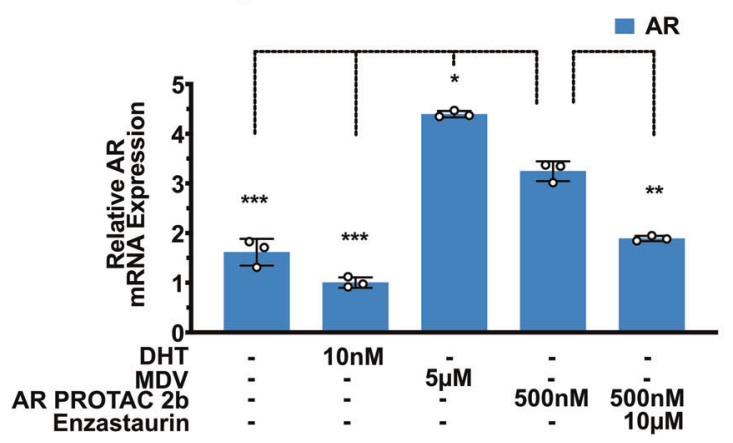

10nM DHT pretreatment, then treated as indicated:

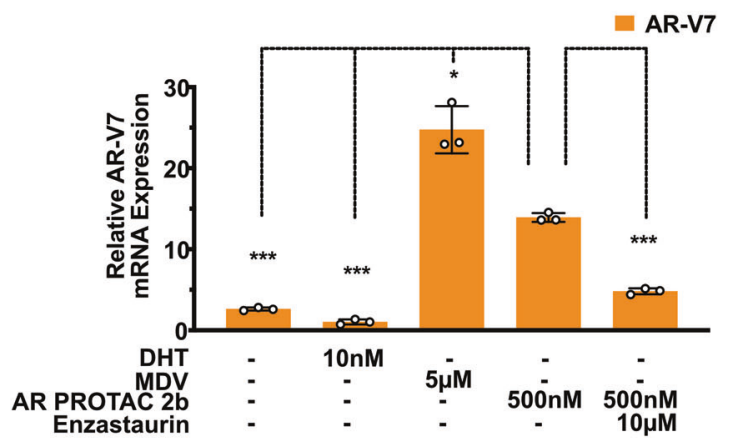

D

\section{Total AR gene expression is building}

$10 \mathrm{nM}$ DHT pretreatment, then treated as indicated:

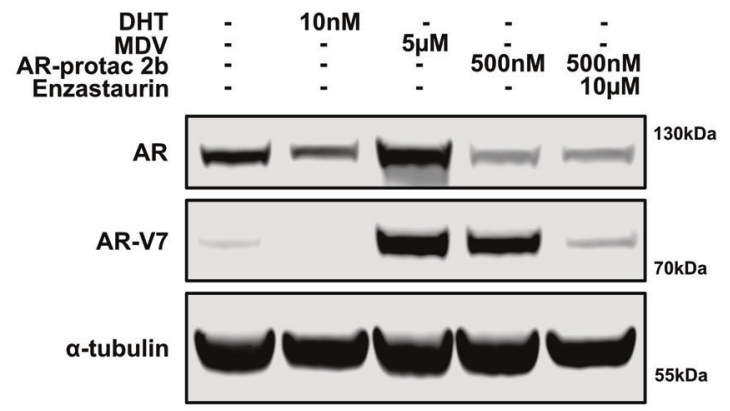

E

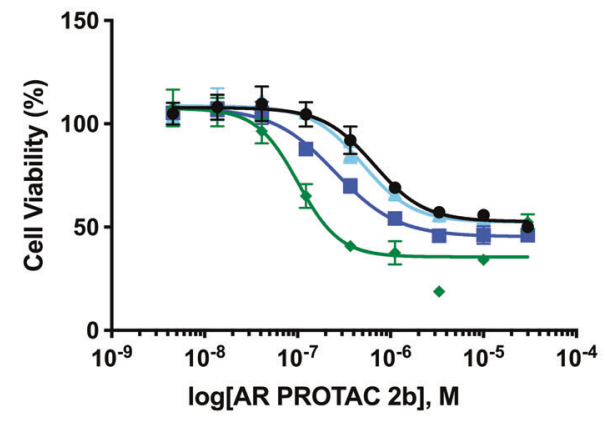

$\rightarrow$ AR PROTAC 2b, IC $50=664 \mathrm{nM}$

$\rightarrow 5 \mu \mathrm{M}$ Enzastaurin + AR PROTAC 2b, IC $50=101 \mathrm{nM}$

- $3 \mu \mathrm{M}$ Enzastaurin + AR PROTAC 2b, IC $50=254 \mathrm{nM}$

$-1 \mu \mathrm{M}$ Enzastaurin + AR PROTAC 2b, IC $50=499 \mathrm{nM}$

Fig. 5 Enzastaurin is compatible with the next-generation AR degraders. A An AR PROTAC consisting of ABM-3 and the VHL ligand connected by a linker. ABM-3 structurally resembles the anti-androgen MDV. B VCaP cells cultured in RPMI 1640 supplemented with $5 \%$ CSS for $48 \mathrm{~h}$ were treated as indicated for $24 \mathrm{~h}$ and then immunoblotted for AR and AR-V7. C VCaP cells cultured in RPMI1640 supplemented with $5 \%$ CSS for $48 \mathrm{~h}$, pre-treated with DHT for $24 \mathrm{~h}$, and then washed out and treated as indicated in three biological replicates for an additional $24 \mathrm{~h}$ and analyzed by RT-qPCR for AR and AR-V7 mRNA transcript levels. Data are mean \pm SD. P-values are relative to AR PROTAC $\mathbf{2 b}\left({ }^{*} p\right.$-value $<$ $0.05 ;{ }^{* *} p$-value $<0.005 ;{ }^{* * *} p$-value $\left.<0.0001\right)$. All mRNA expression levels are relative to GAPDH and normalized to the response for DHT. D VCaP cells cultured in RPMI 1640 supplemented with 5\% CSS for $48 \mathrm{~h}$, pre-treated with DHT for $24 \mathrm{~h}$, and then washed out and treated as indicated for an additional $72 \mathrm{~h}$ and then immunoblotted for AR and AR-V7. E VCaP (5000 cells/well) cells were cultured in RPMI1640 supplemented with $5 \%$ CSS for $48 \mathrm{~h}$ in 96 well plates. In the presence of $0.1 \mathrm{nM} \mathrm{DHT}$, cells were treated with a nine-point threefold dilution series of AR PROTAC $\mathbf{2 b}$ (beginning at $30 \mu \mathrm{M}$ ) in the presence or absence of a constant concentration of Enzastaurin for seven days. Cell viability was subsequently measured in a CellTiter-Glo bioluminescence assay. Data are mean \pm SD ( $N=3$ biological replicates). 
1544

a Bliss Independence model to generate a map of synergistic (red) and antagonistic (green) interactions (Fig. 4B) [45]. We again observed synergy, with the most robust combinations occurring when the concentrations of MDV and enzastaurin are both above $1 \mu \mathrm{M}$. Third, we used CompuSyn 1.0 to calculate Chou-Talalay combination indices (Cls) at different fractional inhibitions ( $\mathrm{Fa}$ ) for a dilution series of our combination in a 1:1 ratio [46, 47]. The Cls reveal synergy across our dilution series, and a sampling of the Cls is presented in Supplementary Table S2.

Next, we assessed our combination by Gaddum's noninteraction in 22RV1 prostate cancer cells. The 22RV1 cells display uninhibited growth in the presence of MDV despite the antagonism of full-length AR androgen-dependent activation, however, knockdown of AR-V7 has been shown to sensitize this cell line to MDV [48]. In our assay, only prolonged treatment of MDV in combination with enzastaurin reveals an inhibitory effect, but could not be fit to a nonlinear regression model (Fig. 4C). Importantly, our data indicate that repression of AR-V7 by enzastaurin does allow for MDV-dependent growth inhibition. Overall, we show that our combination improves the response of AR-V7 positive prostate cancer cells to MDV.

Finally, we assessed our combination by Gaddum's noninteraction in the PC-3 and LNCaP prostate cancer cell lines. The PC-3 cell line is AR-null, and as expected, neither MDV nor our combination of MDV with enzastaurin showed an effect on cell viability (Fig. 4D). The LNCaP cell line contains full-length AR protein with a T878A somatic mutation in its LBD and is resistant to anti-androgens $[49,50]$. LNCaP cells do not express AR-V7 protein, but AR-V7 splice variant mRNA can be detected [5] (Supplementary Fig. S4A and Supplementary Fig. S4B). We observed a weak response for MDV alone and with our combinations in LNCaP cells (Fig. 4E and Supplementary Figure $\mathrm{S} 4 \mathrm{C})$. This response is the most pronounced at higher concentrations of MDV (Supplementary Figure S4C), however, the response achieved in our dose series is not sufficient for the calculation of an $I C_{50}$ value. We suspect this to be due to the resistant nature of the cell line.

\section{Enzastaurin is compatible with next-generation AR degrader strategies in AR-V7 positive prostate cancer cells}

The next generation of AR antagonists, the AR degraders, are in development and under evaluation in clinical trials. These bivalent molecules consist of an anti-androgen tethered to a ligand that recruits an E3 ligase to the full-length $A R$, causing it to be ubiquitinated and targeted for degradation [51-53]. We hypothesized that these molecules will not degrade the AR-V7 splice variant due to the deletion of the LBD. Additionally, degradation of full-length AR protein will ablate AR-dependent gene repression and allow transcriptional activation of the AR genomic locus, thus increasing total AR mRNA transcript levels and potentially driving resistance through AR-V7 (Fig. 1A (middle)). We, therefore, wished to determine if our combination strategy with enzastaurin could effectively reduce AR-V7 splice variant mRNA transcripts in the presence of an AR degrader. First, we synthesized a biologically evaluated AR degrader (AR PROTAC $\mathbf{2 b}$ ) reported previously (Fig. 5A) [53]. This degrader consists of the anti-androgen ABM-3, which structurally resembles MDV, tethered to a ligand that recruits the von Hippel-Lindau (VHL) E3 ligase to the full-length AR causing degradation [54]. We then evaluated AR PROTAC $\mathbf{2 b}$, as well as its separated components, in VCaP cells to confirm that the fulllength AR protein is degraded by the bivalent molecule, but the AR-V7 splice variant protein persists. The bivalent molecule proved highly effective at $500 \mathrm{nM}$. A slight hook effect for full-length AR degradation was observed at $5 \mu \mathrm{M}$, which is an expected effect for bivalent degrader molecules (Fig. 5B) [55].

Having confirmed its efficacy against full-length $A R$, we next evaluated AR PROTAC $\mathbf{2 b}$ in several of our established assays. First, we assessed the VCaP cell line after DHT wash out with AR
PROTAC 2b, an assay in which MDV alleviates AR genomic locus suppression resulting in $A R$ rebound expression. In this experiment, we observed rebound expression of AR and AR-V7 after treatment with AR PROTAC $\mathbf{2 b}$, although the expression was less than that observed with MDV (Fig. 5C). Enzastaurin in combination with AR PROTAC $\mathbf{2 b}$ suppressed AR locus expression and reduced the level of AR and AR-V7 mRNA transcripts by 1.7-fold and 2.9fold respectively, relative to AR PROTAC $\mathbf{2 b}$ alone. The effect was larger when the combination of enzastaurin and AR PROTAC $\mathbf{2 b}$ was compared to MDV, where the relative levels of full-length $A R$ and AR-V7 mRNA transcripts were reduced by 2.3 -fold and 5.1 -fold respectively. We also evaluated AR-V7 protein levels after the same treatments. AR-V7 protein levels when AR PROTAC $\mathbf{2} \mathbf{b}$ and enzastaurin are combined were remarkably lower than AR PROTAC 2b alone (Fig. 5D). Finally, we assessed AR PROTAC 2b in combination with enzastaurin in Gaddum's non-interaction assay, revealing that combination with enzastaurin improves the effectiveness of AR PROTAC 2b (Fig. 5E).

\section{MDV and enzastaurin in combination demonstrate greater efficacy than MDV alone in vivo against VCaP xenografts}

We designed an in vivo study using $\mathrm{VCaP}$ xenografts to mimic reactivation of androgen receptor signaling in CRPC to validate the effectiveness of our combination relative to the MDV monotherapy [56]. Once the VCaP xenografts were established in castrated male mice (Fig. 6A), dosing followed a schedule of five days on and two days off for a total of six weeks with bi-weekly tumor volume measurements. Dosing for the monotherapies was determined from literature precedent [57-59] and dosing for the combination therapy was determined in a tolerability study (Supplementary Fig. S5A). The combination therapy proved more effective than the MDV monotherapy demonstrating that enzastaurin can augment the effect of MDV in vivo (Fig. 6B). Not surprisingly, the enzastaurin monotherapy also proved more effective than the MDV monotherapy due to its ability to suppress total AR gene expression. Upon completion of the study, we analyzed the VCaP xenografts by immunoblot. Our analysis reveals an average reduction in both AR-V7 and histone H3T6 phosphorylation in the combination therapy and enzastaurin monotherapy relative to the MDV monotherapy (Supplementary Fig. S5B, Supplementary Table S3).

\section{DISCUSSION}

CRPC is an aggressive cancer that follows relapse of hormonenaïve prostate cancer. Prognosis is particularly poor when AR-V7 is detected, as ARSi offers little benefit; and patients experience shorter PSA progression-free survival and lower overall survival than patients negative for AR-V7 [3]. The AR-V7 splice variant protein lacks its LBD, and largely consists of an unstructured $\mathrm{N}$-terminal domain and a DNA-binding domain that is highly conserved across the nuclear receptor superfamily [60]. This complicates direct inhibition by a small molecule due to the lack of a 'druggable' pocket that can be targeted with high specificity and selectivity.

Prior work has indicated that the full-length AR auto-regulates its own genomic locus in response to androgen. In a highandrogen environment the full-length AR localizes to intron 2 of the AR genomic locus and recruits LSD1 to remove methyl marks from histone $\mathrm{H} 3 \mathrm{~K} 4$ to suppress transcriptional activity at the locus $[6,31]$. However in a low-androgen environment, AR genomic locus repression is alleviated [6], and transcription at the locus increases $[5,6,9]$. PKC $\beta 1$ is reported to facilitate a subset of transcriptional programs by phosphorylating histone H3T6 to block lysine demethylase activity, thus allowing H3K4 methylation to persist [27]. We hypothesized that in the absence of androgenbound full-length $A R, P K C \beta 1$ is active at the AR genomic locus and promoting transcription. Since spliceosome assembly and pre- 
A

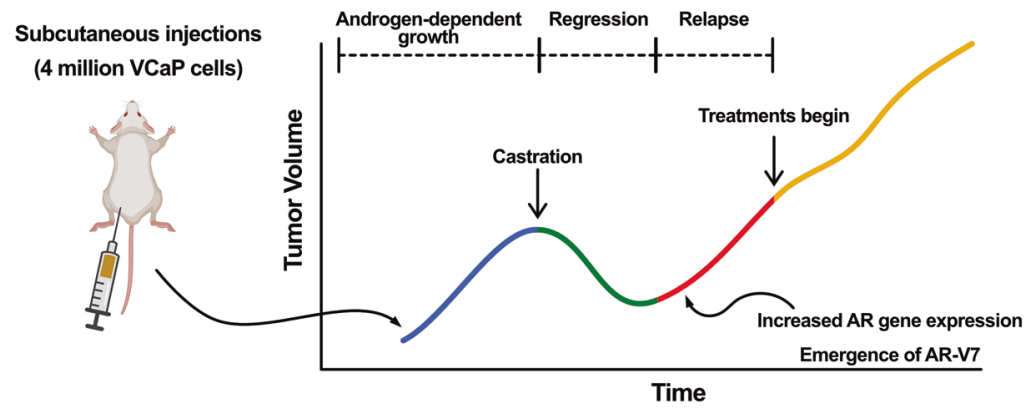

B

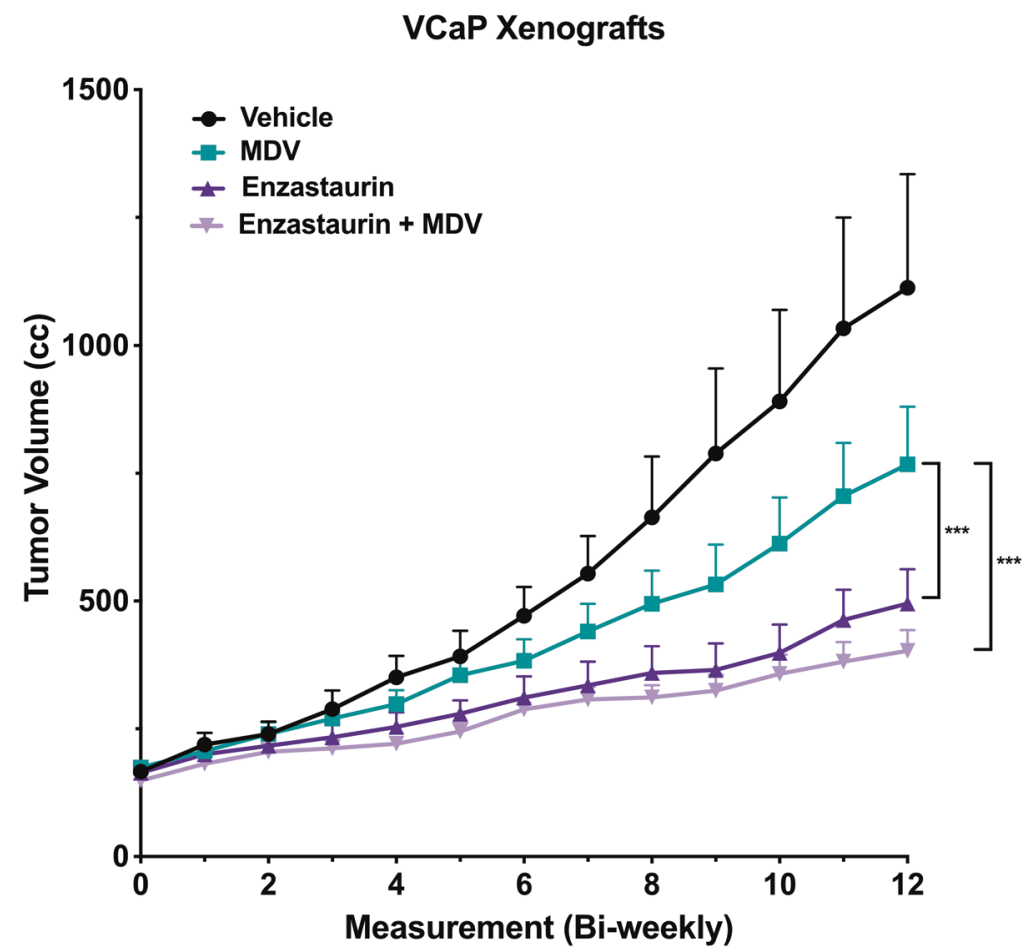

Fig. 6 VCaP xenografts respond positively to Enzastaurin and MDV in combination. A VCaP cells were introduced by subcutaneous flank injection. The xenografts initially developed in mice with circulating androgen. Once tumor volumes reached $\sim 100 \mathrm{cc}$, mice were castrated and the xenografts subsequently shrank until androgen independence was acquired. Xenografts then relapsed and treatments began when tumors reached $\sim 150 \mathrm{cC}$. B Tumor volumes in mice bearing VCaP (AR-V7 positive) xenografts $(N=8-9$ mice per arm). Measurements were recorded biweekly for a total of six weeks. Treatments consisted of vehicle, MDV (10 mg/kg), Enzastaurin (50 mg/kg BID), Enzastaurin (50 mg/ $\mathrm{kg} \mathrm{BID})+\mathrm{MDV}(10 \mathrm{mg} / \mathrm{kg})\left({ }^{* * *} P\right.$-value $\left.<0.0001\right)$. Data are mean \pm SEM.

mRNA processing occurs co-transcriptionally and is dependent on chromatin modifications, we proposed that by employing an epigenetic strategy focused on PKC $\beta 1$ inhibition we could target total AR expression, including AR-V7. We hypothesized that this approach would reduce both full-length $A R$ and the undruggable AR-V7 transcription factor, providing an opportunity to antagonize AR-V7-driven prostate cancer growth. Further, we anticipated that this approach would be amenable to combination with current anti-androgen therapies, allowing co-targeting of AR-V7 and fulllength AR.

We evaluated the clinical PKC $\beta$ inhibitor enzastaurin for its ability to suppress both full-length AR and AR-V7 mRNA transcripts according to our proposed mechanism. Our results indicate that enzastaurin is able to reduce total AR mRNA transcripts, including AR-V7, in the presence of MDV. In particular, we also observed that reductions in $A R-V 7$ mRNA transcripts resulted in a marked reduction in $A R-V 7$ protein levels. Notably, the magnitude of this effect is greater for AR-V7 than it is for full-length AR at both the mRNA and protein levels. We evaluated the kinases targeted by enzastaurin and determined that its effectiveness is dependent on PKC $\beta$ inhibition. Furthermore, the data indicates that GSK3 $a$ and GSK3 $\beta$ inhibition are responsible for a partial reduction in AR-V7 mRNA transcript levels. A number of transcription factors are direct substrates of the GSK3 kinases, and we speculate that the partial effect of GSK3 inhibition may be due to this regulation $[61,62]$. Further, reducing GSK3 activity is reported to sensitize PKC $\beta$ to small molecule inhibitors, therefore another potential mechanism is that co-targeting of GSK3 and PKC $\beta$ by enzastaurin improves the effect of PKC $\beta$ inhibition [63]. We concluded that the effectiveness of enzastaurin is mediated by inhibition of PKC $\beta 1$ and likely enhanced by inhibition of GSK3 $\alpha$ and GSK3 $\beta$ through an additional mechanism.

We used several different synergy models to demonstrate that enzastaurin synergizes with MDV to inhibit AR-V7 positive prostate 
cancer cell growth. The combination exhibited a pronounced effect for cell growth inhibition in AR-V7 positive prostate cancer cell lines. Importantly, no effect was observed for our combinations in other prostate cancer model cell lines that do not express AR-V7. Interestingly, enzastaurin also proved efficacious for reducing $A R-V 7$ splice variant levels in combination with an $A R$ degrader and improved the effectiveness of the degrader. We assessed the effectiveness of our enzastaurin and MDV combination against the MDV monotherapy in a VCaP xenograft mouse model and found that the combination was more effective than MDV alone.

In this work we have attempted to offer a mechanistic explanation for regulation of AR-V7 splicing by our combination therapy. However, protein kinase $C$ regulates a complex signaling network and we recognize that the splicing effects we observe could in part be indirectly regulated through other PKC substrates in addition to our proposed mechanism. Finally, this work was primarily performed in the VCaP cell line, which contains wild-type full-length $A R$ and demonstrates a unique responsiveness to both DHT and MDV compared to other prostate cancer cell lines [5, 6]. Our combination therapy demonstrated the greatest effect in the $\mathrm{VCaP}$ cell line indicating that it may not be as effective in other settings.

To date, enzastaurin has been evaluated in two Phase-Il clinical trials against metastatic CRPC $[64,65]$. In the first trial, patients were grouped into two cohorts: those with progressive nonmetastatic disease and those with progressive metastatic disease following treatment with docetaxel-based chemotherapy. Both cohorts were provided enzastaurin monotherapy. A mild response was observed for patients with progressive metastatic disease [65]. In the second trial, patients with metastatic CRPC received docetaxel with prednisone, with or without enzastaurin. No significant difference was observed between the two groups [64]. At the time of these trials, enzalutamide and other AR antagonists were not considered standard of care therapies, but today, ARSi therapies are far more prevalent and are correlated with a significant increase in the frequency of AR-V7 positive metastatic CRPC $[18,66]$. Further, assays are now clinically available for the detection of AR-V7 positive circulating tumor cells and AR-V7 levels are dictating patient selection for clinical trials $[18,66]$. While the activity of enzastaurin was modest in the pre-ARSi era, due to the establishment of ARSi therapies and the prevalence of AR-V7-positive metastatic CRPC, we feel that our in vitro and in vivo work justifies PKC $\beta 1$ inhibition in combination with $A R$ antagonists as a viable strategy for further clinical evaluation against AR-V7-positive prostate cancer in an AR-V7 biomarker-selected trial. In conclusion, we report a new mechanistic approach based on reducing full-length AR and AR-V7 splice variant protein levels that increases the sensitivity of AR-V7 prostate cancer cells to AR antagonism.

\section{METHODS}

\section{Cell culture and reagents}

All cell lines in this study (VCaP, CRL-2876; PC-3, CRL-1435; LNCaP, CRL1740) were obtained from the American Type Culture Collection (ATCC), with the exception of the 22RV1 cell line, which was provided to us by Felix Feng. VCaP, PC-3 and LNCaP cells were cultured in $5 \% \mathrm{CO}_{2}$ at $37^{\circ} \mathrm{C}$ with Dulbecco's modified Eagle's medium (DMEM) (ATCC, 30-2002) supplemented with $10 \%(\mathrm{v} / \mathrm{v})$ heat-inactivated fetal bovine serum (Axenia BioLogix). 22RV1 cells were cultured in $5 \% \mathrm{CO}_{2}$ at $37^{\circ} \mathrm{C}$ with Roswell Park Memorial Institute medium (RPMI) (ATCC, 30-2001) supplemented with $10 \%(\mathrm{v} / \mathrm{v})$ heat-inactivated fetal bovine serum (Axenia BioLogix). Cellular assays were performed in RPMI medium supplemented with $5 \%(\mathrm{v} / \mathrm{v})$ charcoal stripped fetal bovine serum (CSS) (Gibco, A33821). Cells were periodically tested for contamination using the MycoAlert Plus Mycoplasma Detection Kit (Lonza). Dihydrotestosterone was purchased from Sigma Aldrich. Enzalutamide (MDV) was purchased from MedChem Express. Enzastaurin was purchased from both Selleck Chemicals and MedChem Express. All reagents used for synthesis of the AR degrader were obtained from Sigma Aldrich, Acros Organics, Cayman Chemicals or AstaTech. The AR degrader was synthesized as described [53].

\section{SDS-PAGE and Immunoblotting}

Treated cells $(\sim 500,000-1,000,000$ cells/well) were lysed with RIPA buffer or with $20 \mathrm{mM}$ Tris- $\mathrm{HCl}, \mathrm{pH} 7.5,0.5 \mathrm{mM}$ EDTA, $0.5 \mathrm{mM}$ EGTA, $1 \mathrm{mM}$ DTT, $10 \%$ Glycerol and $0.5 \%$ IGEPAL CA-630 supplemented with phosphate inhibitors (Roche, PhosSTOP) and protease inhibitors (Roche, cOmplete Protease Inhibitor Cocktail Tablets), and protein concentration was determined by either a Bradford Assay (Bio-Rad, Protein Assay Dye Reagent Concentrate) or a bicinchoninic acid assay (Thermo Fisher Scientific, Pierce BCA Protein Assay Kit). Protein lysates were resolved by SDS-PAGE, transferred to nitrocellulose membranes (Bio-Rad) and blocked using either $5 \%$ milk or $5 \%$ BSA in TBST buffer (1X Tris-buffered saline (TBS), $0.1 \%$ Tween-20). Nitrocellulose membranes were immunoblotted with antibodies against AR (1:1000 in 5\% milk/TBST; Santa Cruz Biotech, sc7305), AR-V7 (1:500 5\% milk/TBST; Precision Antibody, AG-10008); PKC 1 (1:500 in 5\% Milk/TBST; Abcam, ab195039), H3T6ph antibody (1:500 in 5\% BSA/TBST; Abcam, ab222768), Histone H3 (1:2000 5\% BSA/TBST; Cell Signaling Technology, 4499), a-tubulin (1:1000 in 5\% BSA/TBST; Cell Signaling Technology, 3873) and GAPDH $(1: 1000$ in 5\% BSA/TBST; Proteintech, 60004-1-lg). Following the primary antibodies, nitrocellulose membranes were incubated with IRDye secondary antibodies (LI-COR Biosciences) and analyzed on an Odyssey Imaging System (LI-COR Biosciences) according to manufacturer instructions.

\section{qRT-PCR analysis}

RNA from cells $(\sim 500,000$ cells/well) treated in biological replicates $(N=3)$ were isolated using the RNeasy Plus Mini Kit (QIAGEN) according to manufacturer instructions. Reverse transcription was performed using the SuperScript III First-Strand Synthesis SuperMix for qRT-PCR (Life Technologies) according to manufacturer instructions. The reverse transcription products were evaluated by qRT-PCR using the Maxima SYBR Green qPCR Master Mix (Life Technologies) on a Bio-Rad CFX Touch Real-Time PCR system according to manufacturer instructions. GAPDH served as a reference gene. All samples were evaluated using the $\Delta \Delta \mathrm{Cq}$ method under the gene expression tab in the Bio-Rad CFX Maestro for Mac 1.1 software. Primer sequences are as follows: AR forward: $5^{\prime}$-TCT TGT CGT CTT CGG AAA TGT-3', AR reverse: 5'-AAG CCT CTC CTT CCT CCT GTA-3'; ARV7 forward: 5'-CAG GGA TGA CTC TGG GAG AA-3', AR-V7 reverse: 5'-GCC CTC TAG AGC CCT CAT TT-3'; GAPDH forward: 5'-GGA CCT GAC CTG CCG TCT AG AA-3', GAPDH reverse: 5'-GGT GTC GCT GTT GAA GTC AGA G-3'; MCM2 forward: 5'-ATT TCG TCC TGG GTC CTT TC-3', MCM2 reverse: 5'-GCT GGT AGT TCT GAT AGA TGG T-3'; MCM7 forward: 5'-GGA TGC CAC CTA TAC TTC TGC-3', MCM7 reverse: 5'-CCT TTG ACA TCT CCA TTA GCC T-3'; FANCI forward: 5'-CAA TGA GGA ACA GAG TGG TGA-3', FANCl reverse: 5'-GCC TAG TTC ATA GTC CAA TाT GAT G-3'; LMNB1 forward: 5'-GGA AAT CAG TGC TTA CAG GAA AC-3', LMNB1 reverse: 5'-CTT GAG GAT GCT CGG GAT AC-3'; RAD51AP1 forward: 5'-GTC TTC AGA TAC CAC TAG GAA ACC-3'; RAD51AP1 reverse: 5'-CTG CTG CTA CTT CTG CTA CC-3'; OPRK1 forward: 5'-TCA TCA ATA TCT GCA TCT GGC T-3'; OPRK1 reverse: 5'-AAG GAG CAC TCA ATG ACA TCG-3'; AKR1C3 forward: 5'-GGC CAC TTC ATG CCT GTA-3', AKR1C3 reverse: 5'-GAA CCC AGC TTC TAT TGC TAA-3'.

\section{Multiplexed inhibitor beads (MIB) assay}

Kinase chromatography, mass spectrometry and analytical processing were performed as described previously [40]. Briefly, cells growing in RPMI 1640 (ATCC, 30-2001) supplemented with $5 \%(\mathrm{v} / \mathrm{v})$ charcoal-stripped fetal bovine serum (CSS) (Gibco, A33821) for $48 \mathrm{~h}$ were treated in three biological replicates for $24 \mathrm{~h}$ with DMSO, DHT, or MDV and then collected in PBS. Samples were lysed in $150 \mathrm{mM} \mathrm{NaCl}$ buffer with protease and phosphatase inhibitors, and then diluted in $1 \mathrm{M} \mathrm{NaCl}$ binding buffer. Affinity purification was performed with gravity chromatography after preclearing. The bound kinases were washed and eluted followed by extraction/precipitation, tryptic digest, and desalting. Liquid chromatography-tandem mass spectrometry (LC/MS-MS) was performed on a Q-Exactive with in-line high-performance liquid chromatography (HPLC) at the Thermo Fisher Scientific Proteomics Facility for Disease Target Discovery at UCSF and the J. David Gladstone Institutes. Peptide identification was done with MaxQuant, label-free quantification with Skyline [67], and statistical analysis with MSstat [68]. 


\section{ChIP qRT-PCR assay}

Cells growing in 10 or $15-\mathrm{cm}$ plates were cultured in RPMI 1640 (ATCC, 302001) supplemented with $5 \%(\mathrm{v} / \mathrm{v})$ charcoal stripped fetal bovine serum (CSS) (Gibco, A33821) for $48 \mathrm{~h}$. Plates were then treated in biological replicates with vehicle, $5 \mu \mathrm{M}$ MDV or $5 \mu \mathrm{M}$ MDV $+10 \mu \mathrm{M}$ Enzastaurin for $24 \mathrm{~h}$. Samples were subsequently processed using the Zymo-Spin ChIP Kit (D5209) and either a H3T6ph antibody (Abcam, ab222768), H3K4Me2 (Cell Signaling Technology, 9725), H3K4Me1 (Cell Signaling Technology, 5326), LSD1 (Abcam, 129195) or a rabbit IgG antibody (Cell Signaling Technology, 2729). The precipitated DNA was evaluated by qRT-PCR using the Maxima SYBR Green qPCR Master Mix (Life Technologies) on a Bio-Rad CFX Touch Real-Time PCR system according to manufacturer instructions. Data is reported as percent of input. Primer sequences are as follows: ARBS2d' forward: GCT CAG AGA GGT TाT AGT TGT G, ARBS2d' reverse: CAA AAT GTC TAA GCT GGA AGC AC; ARBS2 $b^{\prime}$ forward: GTC TTG CTT TCC TAG AAG GTG AC; ARBS2b' reverse: CAA GGA GAA AAT CTG AGT CCT GAG; ARBS2b forward: CAC ATG GAG TGC TGT TTG GT, ARBS2b reverse: GTA AAC ATC AGT GAG GAT GGT G; ARBS2e forward: GCA GAG AGT TाT TGG TGC ATA TC, ARBS2e reverse: CAA AGA TAC CTG ATG AAG GCT CTG; ARBS2g forward: CAG ACT TTA GAT TTA GGG GTT GG, ARBS2g reverse: GTC TAT GGC TGC TIT CAT CCT AC.

\section{Drug synergy assays}

Cells were seeded into white 96-well clear flat bottom plates (Corning, 3903) in RPMI 1640 (ATCC, 30-2001) supplemented with 5\% (v/v) charcoal stripped fetal bovine serum (CSS) (Gibco, A33821) for $48 \mathrm{~h}$. Cells were then treated in biological replicates $(N=3)$ accordingly: Gaddum's noninteraction: In the presence of $0.1 \mathrm{nM}$ DHT, MDV in a nine-point threefold dilution series at Enzastaurin concentrations of $5 \mu \mathrm{M}, 3 \mu \mathrm{M}, 1 \mu \mathrm{M}$ or $0 \mu \mathrm{M}$; Checkerboard assay: In the presence of $0.1 \mathrm{nM}$ DHT, MDV and Enzastaurin checkerboarded in a threefold dilution series; Chou-Talalay combination indices: In the presence of $0.1 \mathrm{nM}$ DHT, MDV and Enzastaurin as monotherapies or as a 1:1 concentration ratio combination in a ninepoint threefold dilution series. Cell viability was assessed after three or five days using a CellTiter-Glo luminescence-based assay (Promega). The CellTiter-Glo reagent was diluted fivefold in PBS and added to cells in a 1:1 ratio with the cellular growth medium. Plates were incubated with shaking at room temperature for $20 \mathrm{~min}$ and then the luminescence signal was recorded on a Tecan Spark plate reader. Bliss synergy scores were calculated using https://synergyfinder.fimm.fi/. Chou-Talalay combination indices were determined using CompuSyn 1.0 (negative viability measurements were substituted with a value of 0.0001 ).

\section{VCaP xenograft study and preparation for Immunoblotting}

All mouse manipulations were performed in accordance with the University of California, San Francisco's Institutional Animal Care and Use Committee. All animals were housed in specific pathogen-free conditions and cared for according to the International Association for Assessment and Accreditation of Laboratory Animal Care policies and certification (IACUC protocol ANI179937). All surgeries were performed under isoflurane anesthesia. Six- to eight-week-old male NSG mice (005557, Jackson Lab, Bar Harbor, ME) were bred in house and housed with ad libitum food and water on a $12 \mathrm{~h}$ light cycle at the UCSF Preclinical Therapeutics Core vivarium. VCaP xenografts were introduced to the right flanks of mice by subcutaneous injection (4 million cells in $100 \mu \mathrm{L} ; 1: 1$ ratio of Corning Matrigel and serum-free DMEM). Mice were castrated on a rolling basis as the VCaP xenografts reached sizes of $\sim 100 \mathrm{~mm}^{3}$. The xenografts would shrink following castration, and then regrow. Mice were enrolled evenly and randomly into each arm of the study on a rolling basis as the xenografts approached sizes of $\sim 150 \mathrm{~mm}^{3}$. Mice were dosed by oral gavage on a schedule five days on followed by two days off. Arms consisted of: Vehicle ( $1 \%$ carboxymethyl cellulose, $0.1 \%$ Tween- $80,5 \%$ DMSO), MDV - $10 \mathrm{mg} / \mathrm{kg}$ (1\% carboxymethyl cellulose, $0.1 \%$ Tween-80, $5 \%$ DMSO), Enzastaurin - $50 \mathrm{mg} / \mathrm{kg}$ BID (5\% DMSO, 15\% Captisol) and MDV + Enzastaurin. Tumor volumes and body weights were collected biweekly over the course of the six week study. Tumor volumes were assessed by $2 \mathrm{D}$ caliper measurements and volume was calculated according to the volume of an ellipsoid $\left(V=0.52 \times\right.$ (width $^{2} \times$ length). The xenografts were collected and flash frozen upon termination of the time course study. Pieces of the xenografts were crushed under liquid nitrogen, and the tissue was lysed with RIPA buffer containing $1 \mathrm{X}$ PhosSTOP, $1 \mathrm{X}$ PIC and $1 \mathrm{mM}$ PMSF on ice with occasional vortexing. Samples were analyzed according the protocol outlined in the SDS Page and Immunoblotting section.

\section{Statistical analysis}

qRT-PCR $\Delta \Delta$ Ct values were calculated using the Bio-Rad CFX Maestro software and then plotted using Graphpad Prism 8 as the mean \pm SD with individual data points shown, and the Tukey $P$-values are reported from the ANOVA tab. Immunoblots were processed with Image Studio Lite 5.2.5 (LI-COR). Gaddum's non-interaction data is represented as mean $\pm S D$, and $\mathrm{IC}_{50}$ values were determined in Graphpad Prism 8 using a log(inhibitor) vs response - variable slope (four parameter) model. The Bliss synergy grid was modeled using https://synergyfinder.fimm.fi/. Chou-Talalay combination indices were calculated using CompuSyn 1.0. P-values for ChIP-qPCR assay calculated in Microsoft Excel using the two-tail Student's T-test function assuming equal variance. P-values for the xenograft studies were calculated by two-way ANOVA in Graphpad Prism 8.

\section{DATA AVAILABILITY}

The full immunoblot images are provided in Supplementary Fig. S6. All data generated or analyzed during the current study are included in this published article.

\section{REFERENCES}

1. Kirby $M$, Hirst $C$, Crawford ED. Characterising the castration-resistant prostate cancer population: A systematic review. Int J Clin Pr. 2011;65:1180-92.

2. Heinlein $C A$, Chang C.Androgen receptor in prostate cancer. Endocr Rev. 2004;25:276-308.

3. Antonarakis ES, Lu C, Wang H, Luber B, Nakazawa M, Roeser JC, et al. AR-V7 and resistance to enzalutamide and abiraterone in prostate cancer. N. Engl J Med. 2014;371:1028-38.

4. Quigley DA, Dang HX, Zhao SG, Lloyd P, Aggarwal R, Alumkal JJ, et al. Genomic hallmarks and structural variation in metastatic prostate cancer. Cell. 2018;174:758-9.

5. Liu LL, Xie N, Sun S, Plymate S, Mostaghel E, Dong X. Mechanisms of the androgen receptor splicing in prostate cancer cells. Oncogene. 2014;33:3140-50.

6. Cai C, He HH, Chen S, Coleman I, Wang H, Fang Z, et al. Androgen receptor gene expression in prostate cancer is directly suppressed by the androgen receptor through recruitment of Lysine-specific Demethylase 1. Cancer Cell. 2011;20:457-71.

7. Hu R, Dunn TA, Wei S, Isharwal S, Veltri RW, Humphreys E, et al. Ligandindependent androgen receptor variants derived from splicing of cryptic exons signify hormone-refractory prostate cancer. Cancer Res. 2009;69:16-22.

8. Hu R, Lu C, Mostaghel EA, Yegnasubramanian S, Gurel M, Tannahill C, et al. Distinct transcriptional programs mediated by the ligand-dependent full-length androgen receptor and its splice variants in castration-resistant prostate cancer. Cancer Res. 2012;72:3457-62.

9. Yu Z, Chen S, Sowalsky AG, Voznesensky OS, Mostaghel EA, Nelson PS, et al. Rapid induction of androgen receptor splice variants by androgen deprivation in prostate cancer. Clin Cancer Res. 2014;20:1590-1600.

10. Yang Z, Wang D, Johnson JK, Pascal LE, Takubo K, Avula R, et al. A novel small molecule targets androgen receptor and its splice variants in castration-resistant prostate cancer. Mol Cancer Ther. 2020;19:75-88.

11. Myung J-K, Banuelos CA, Fernandez JG, Mawji NR, Wang J, Tien AH, et al. An androgen receptor $\mathrm{N}$-terminal domain antagonist for treating prostate cancer. J Clin Invest. 2013;123:2948-60.

12. Mol ED, Fenwick RB, Phang CTW, Buzón V, Szulc E, de la Fuente A, et al. EPI-001, A compound active against castration-resistant prostate cancer, targets transactivation unit 5 of the androgen receptor. Acs Chem Biol. 2016;11:2499-505.

13. Li H, Ban F, Dalal K, Leblanc E, Frewin K, Ma D, et al. Discovery of small-molecule inhibitors selectively targeting the dna-binding domain of the human androgen receptor. J Med Chem. 2014;57:6458-67.

14. Liu C, Lou W, Zhu Y, Nadiminty N, Schwartz CT, Evans CP, et al. Niclosamide inhibits androgen receptor variants expression and overcomes enzalutamide resistance in castration-resistant prostate cancer. Clin Cancer Res. 2014;20:3198-210.

15. Kwegyir-Afful AK, Ramalingam S, Tamachar PP, Ramamurthy VP, Njar VCO. Galeterone and VNPT55 induce proteasomal degradation of AR:AR-V7, induce significant apoptosis via cytochrome $\mathrm{c}$ release and suppress growth of castration resistant prostate cancer xenografts in vivo.pdf. Oncotarget. 2015;6:27440-60.

16. Melnyk JE, Steri V, Nguyen HG, Hann B, Feng FY, Shokat KM. The splicing modulator sulfonamide indisulam reduces AR-V7 in prostate cancer cells. Bioorgan Med Chem. 2020;28:115712.

17. Khurana N, Chandra PK, Kim H, Abdel-Mageed AB, Mondal D, Sikka SC. Bardoxolone-Methyl (CDDO-Me) suppresses androgen receptor and its splicevariant AR-V7 and enhances efficacy of Enzalutamide in prostate cancer cells. Antioxidants. 2020;9:68. 
18. Armstrong CM, Gao AC. Current strategies for targeting the activity of androgen receptor variants. Asian J Urol. 2019;6:42-49.

19. Zhang X, Castanotto D, Nam S, Horne D, Stein C. $6 B I O$ enhances oligonucleotide activity in cells: A potential combinatorial anti-androgen receptor therapy in prostate cancer cells. Mol Ther. 2017;25:79-91.

20. Velez MVL, Verhaegh GW, Smit F, Sedelaar JPM, Schalken JA. Suppression of prostate tumor cell survival by antisense oligonucleotide-mediated inhibition of AR-V7 mRNA synthesis. Oncogene. 2019;38:3696-709.

21. Velasco MAD, Kura Y, Sakai K, Hatanaka Y, Davies BR, Campbell H, et al. Targeting castration-resistant prostate cancer with androgen receptor antisense oligonucleotide therapy. Jci Insight. 2019;4:e122688.

22. Carabet LA, Leblanc E, Lallous N, Morin H, Ghaidi F, Lee J, et al. Computer-aided discovery of small molecules targeting the rna splicing activity of hnrnp a1 in castration-resistant prostate cancer. Molecules. 2019;24:763.

23. Bianchini D, Omlin A, Pezaro C, Lorente D, Ferraldeschi R, Mukherji D, et al. Firstin-human Phase I study of EZN-4176, a locked nucleic acid antisense oligonucleotide to exon 4 of the androgen receptor mRNA in patients with castrationresistant prostate cancer. Brit J Cancer. 2013;109:2579-86.

24. Xiao L, Tien JC, Vo J, Tan M, Parolia A, Zhang Y, et al. Epigenetic reprogramming with antisense oligonucleotides enhances the effectiveness of androgen receptor inhibition in castration-resistant prostate cancer. Cancer Res. 2018; 78: canres.0941.2018.

25. Metzger E, Wissmann M, Yin N, Müller JM, Schneider R, Peters AHFM, et al. LSD1 demethylates repressive histone marks to promote androgen-receptordependent transcription. Nature. 2005;437:436-9.

26. Metzger E, Yin N, Wissmann M, Kunowska N, Fischer K, Friedrichs $N$, et al. Phosphorylation of histone $\mathrm{H} 3$ at threonine 11 establishes a novel chromatin mark for transcriptional regulation. Nat Cell Biol. 2008;10:53-60.

27. Metzger E, Imhof A, Patel D, Kahl P, Hoffmeyer K, Friedrichs N, et al. Phosphorylation of histone $\mathrm{H} 3 \mathrm{~T} 6$ by $\mathrm{PKC} \beta \mathrm{I}$ controls demethylation at histone H3K4. Nature. 2010;464:792-6.

28. Metzger E, MuÈller JM, Ferrari S, Buettner R, SchuĖle R. A novel inducible transactivation domain in the androgen receptor- implications for PRK in prostate cancer.pdf. EMBO J. 2003;22:270-80.

29. Gao S, Gao Y, He HH, Han D, Han W, Avery A, et al. Androgen receptor tumor suppressor function is mediated by recruitment of retinoblastoma protein. Cell Rep. 2016;17:966-76.

30. Han D, Chen S, Han W, Gao S, Owiredu JN, Li M, et al. ZBTB7A mediates the transcriptional repression activity of the androgen receptor in prostate cancer. Cancer Res. 2019;79:5260-71.

31. Cai C, He HH, Gao S, Chen S, Yu Z, Gao Y, et al. Lysine-specific Demethylase 1 has dual functions as a major regulator of androgen receptor transcriptional activity. Cell Rep. 2014;9:1618-27.

32. Makkonen $H$, Kauhanen $M$, Jääskeläinen $T$, Palvimo JJ. Androgen receptor amplification is reflected in the transcriptional responses of vertebral-cancer of the prostate cells. Mol Cell Endocrinol. 2011;331:57-65.

33. Shiota M, Yokomizo A, Takeuchi A, Imada K, Kashiwagi E, Song Y, et al. Inhibition of protein kinase $\mathrm{C} / \mathrm{T}$ wist1 signaling augments anticancer effects of androgen deprivation and enzalutamide in prostate cancer. Clin Cancer Res. 2014;20:951-61.

34. Klaeger S, Heinzlmeir S, Wilhelm M, Polzer H, Vick B, Koenig P-A, et al. The target landscape of clinical kinase drugs. Science. 2017;358:eaan4368.

35. Leontieva OV, Black JD. Identification of two distinct pathways of protein kinase ca down-regulation in intestinal epithelial cells*. J Biol Chem. 2004;279:5788-801.

36. Huang FL, Yoshida Y, Cunha-Melo JR, Beaven MA, Huang KP. Differential downregulation of protein Kinase C isozymes. J Biol Chem. 1989;264:4238-43.

37. Newton AC. Protein kinase C: poised to signal. Am J Physiol-Endoc M. 2010;298: E395-E402.

38. Hansra G, Garcia-paramio P, Prevostel C, Whelan RDH, Bornancin F, Parker PJ. Multisite dephosphorylation and desensitization of conventional protein kinase $C$ isotypes. Biochem J. 1999;342:337-44.

39. Putney JW, Tomita T. Phospholipase C signaling and calcium influx. Adv Biol Regul. 2012;52:152-64

40. Saha SK, Gordan JD, Kleinstiver BP, Vu P, Najem MS, Yeo J-C, et al. Isocitrate dehydrogenase mutations confer dasatinib hypersensitivity and SRC dependence in intrahepatic cholangiocarcinoma. Cancer Disco. 2016;6:727-39.

41. Li Y, Hwang TH, Oseth LA, Hauge A, Vessella RL, Schmechel SC, et al. AR intragenic deletions linked to androgen receptor splice variant expression and activity in models of prostate cancer progression. Oncogene. 2012;31:4759-67.

42. Li Y, Alsagabi M, Fan D, Bova GS, Tewfik AH, Dehm SM. Intragenic rearrangement and altered RNA splicing of the androgen receptor in a cell-based model of prostate cancer progression. Cancer Res. 2011;71:2108-17.

43. Lehár J, Zimmermann GR, Krueger AS, Molnar RA, Ledell JT, Heilbut AM, et al. Chemical combination effects predict connectivity in biological systems. Mol Syst Biol. 2007;3:80.
44. Yadav B, Wennerberg K, Aittokallio T, Tang J. Searching for drug synergy in complex dose-response landscapes using an interaction potency model. Comput Struct Biotechnol J. 2015;13:504-13.

45. Aleksandr I, He L, Aittokallio T, Tang J. SynergyFinder: A web application for analyzing drug combination dose-response matrix data. Bioinformatics. 2017; 33: btx162.

46. Chou T-C. Theoretical basis, experimental design, and computerized simulation of synergism and antagonism in drug combination studies. Pharm Rev. 2006;58:621-81.

47. Chou T-C. Drug combination studies and their synergy quantification using the Chou-Talalay method. Cancer Res. 2010;70:440-6.

48. Li Y, Chan SC, Brand L, Hwang TH, Silverstein KAT, Dehm SM. Androgen receptor splice variants mediate enzalutamide resistance in castration-resistant prostate cancer cell lines. Cancer Res. 2013;73:483-9.

49. Watson PA, Arora VK, Sawyers CL. Emerging mechanisms of resistance to androgen receptor inhibitors in prostate cancer. Nat Rev Cancer. 2015;15:701-11.

50. Prekovic S, Royen ME, van, Voet ARD, Geverts B, Houtman R, Melchers $D$, et al. The Effect of F877L and T878A mutations on androgen receptor response to enzalutamide. Mol Cancer Ther. 2016;15:1702-12.

51. Hwang D-J, He Y, Ponnusamy S, Mohler ML, Thiyagarajan T, McEwan IJ, et al. New generation of selective androgen receptor degraders: Our initial design, synthesis, and biological evaluation of new compounds with enzalutamide-resistant prostate cancer activity. J Med Chem. 2018;62:491-511.

52. Ponnusamy S, He Y, Hwang D-J, Thiyagarajan T, Houtman R, Bocharova V, et al. Orally bioavailable androgen receptor degrader, potential next-generation therapeutic for enzalutamide-resistant prostate cancer. Clin Cancer Res. 2019;25:6764-80.

53. Salami J, Alabi S, Willard RR, Vitale NJ, Wang J, Dong H, et al. Androgen receptor degradation by the proteolysis-targeting chimera ARCC-4 outperforms enzalutamide in cellular models of prostate cancer drug resistance. Commun Biol. 2018;1:100.

54. Beretta GL, Zaffaroni N. Androgen receptor-directed molecular conjugates for targeting prostate cancer. Front Chem. 2019;7:369.

55. An S, Fu L. Small-molecule PROTACs: An emerging and promising approach for the development of targeted therapy drugs. Ebiomedicine. 2018;36:553-62.

56. Loberg RD, St John LN, Day LL, Neeley CK, Pienta KJ. Development of the VCaP androgen independent model of prostate cancer. Urol Oncol. 2006;24:161-8.

57. Tran C, Ouk S, Clegg NJ, Chen Y, Watson PA, Arora V, et al. Development of a second-generation antiandrogen for treatment of advanced prostate cancer. Science. 2009;324:787-90.

58. Graff JR, McNulty AM, Hanna KR, Konicek BW, Lynch RL, Bailey SN, et al. The protein kinase $C \beta$-Selective Inhibitor, Enzastaurin (LY317615. $\mathrm{HCl}$ ), suppresses signaling through the AKT pathway, induces apoptosis, and suppresses growth of human colon cancer and glioblastoma xenografts. Cancer Res. 2005;65:7462-9.

59. Podar K, Raab MS, Zhang J, McMillin D, Breitkreutz I, Tai Y-T, et al. Targeting PKC in multiple myeloma- in vitro and in vivo effects of the novel, orally available small-molecule inhibitor enzastaurin (LY317615.HCl). Blood. 2007;109:1669-76.

60. Bain DL, Heneghan AF, Connaghan-Jones KD, Miura MT. Nuclear receptor structure: Implications for function. Physiology. 2007;69:201-20.

61. Beurel E, Grieco SF, Jope RS. Glycogen synthase kinase-3 (GSK3): Regulation, actions, and diseases. Pharm Therapeut. 2015;148:114-31.

62. Maurer U, Preiss F, Brauns-Schubert P, Schlicher L, Charvet C. GSK-3 - at the crossroads of cell death and survival. J Cell Sci. 2014;127:1369-78.

63. Thorne CA, Wichaidit C, Coster AD, Posner BA, Wu LF, Altschuler SJ. GSK-3 modulates cellular responses to a broad spectrum of kinase inhibitors. Nat Chem Biol. 2015;11:58-63.

64. Dreicer R, Garcia J, Rini B, Vogelzang N, Srinivas S, Somer B, et al. A randomized, double-blind, placebo-controlled, Phase II study with and without enzastaurin in combination with docetaxel-based chemotherapy in patients with castrationresistant metastatic prostate cancer. Invest N. Drug. 2013;31:1044-50.

65. Dreicer R, Garcia J, Hussain M, Rini B, Vogelzang N, Srinivas S, et al. Oral enzastaurin in prostate cancer: A two-cohort phase II trial in patients with PSA progression in the non-metastatic castrate state and following docetaxel-based chemotherapy for castrate metastatic disease. Invest N. Drug. 2011;29:1441-8.

66. Scher HI, Lu D, Schreiber NA, Louw J, Graf RP, Vargas HA, et al. Association of ARV7 on circulating tumor cells as a treatment-specific biomarker with outcomes and survival in castration-resistant prostate cancer. Jama Oncol. 2016;2:1441.

67. Schilling B, Rardin MJ, MacLean BX, Zawadzka AM, Frewen BE, Cusack MP, et al. Platform-independent and label-free quantitation of proteomic data using MS1 extracted ion chromatograms in skyline APPLICATION TO PROTEIN ACETYLATION AND PHOSPHORYLATION*. Mol Cell Proteom. 2012;11:202-14.

68. Choi M, Chang C-Y, Clough T, Broudy D, Killeen T, MacLean B, et al. MSstats: An R package for statistical analysis of quantitative mass spectrometry-based proteomic experiments. Bioinformatics. 2014;30:2524-6. 


\section{ACKNOWLEDGEMENTS}

We thank Davide Ruggero, the UCSF Preclinical Therapeutics Core and the UCSF/ Gladstone Institutes Thermo Fisher Scientific Proteomics Facility for research assistance and discussion. We thank Amy Crossan, Shizong A. Dai, Douglas Wassarman, and Jack Stevenson for critical reading of this paper. Portions of Fig. 1A and Fig. 6A were created with BioRender.com. This work is supported by NIH grant 1F32CA236347-01 (to J.E.M.); PCF YI and Challenge grants (to H.G.N.); Burroughs Wellcome Fund Career Award (to J.D. G.); NIH R01 CA227025 and the Benioff Initiative for Prostate Cancer Research (to F.Y.F.), the Howard Hughes Medical Institute, Samuel Waxman Cancer Research Foundation, NIH 1R01CA221969-01 and NIH 1R01CA244550 (to K.M.S.)

\section{AUTHOR CONTRIBUTIONS}

Conception and design: JEM, KMS. Development of methodology: JEM, VS, BH, FYF, and KMS. Acquisition of data (provided animals, acquired and managed patients, provided facilities, etc.): JEM, VS, YCH, HGN, JDG, BH, FYF, and KMS. Analysis and interpretation of data (e.g., statistical analysis, biostatistics, computational analysis): JEM, YCH, and JDG. Writing, review, and/or revision of the paper: JEM, VS, YCH, HGN, JDG, BH, FYF, and KMS. Administrative, technical, or material support (i.e., reporting or organizing data, constructing databases): JEM, YCH, and JDG. Study supervision: KMS.

\section{COMPETING INTERESTS}

KMS has consulting agreements for the following companies involving cash and/or stock compensation: Black Diamond Therapeutics, BridGene Biosciences, Denali Therapeutics, eFFECTOR Therapeutics, Erasca, Genentech/Roche, Janssen Pharmaceuticals, Kumquat Biosciences, Kura Oncology, Merck, Mitokinin, Petra Pharma, Revolution Medicines, Type6 Therapeutics, Venthera, Wellspring Biosciences (Araxes Pharma). FYF has received consulting fees from Astellas, Bayer, Blue Earth Diagnostics, Celgene, Genentech, Janssen, Myovant, Roivant, and Sanofi. No potential conflicts of interest were disclosed by the other authors.

\section{ADDITIONAL INFORMATION}

Supplementary information The online version contains supplementary material available at https://doi.org/10.1038/s41388-022-02179-z.

Correspondence and requests for materials should be addressed to Kevan M. Shokat

Reprints and permission information is available at http://www.nature.com/ reprints

Publisher's note Springer Nature remains neutral with regard to jurisdictional claims in published maps and institutional affiliations.

(i) Open Access This article is licensed under a Creative Commons Attribution 4.0 International License, which permits use, sharing, adaptation, distribution and reproduction in any medium or format, as long as you give appropriate credit to the original author(s) and the source, provide a link to the Creative Commons license, and indicate if changes were made. The images or other third party material in this article are included in the article's Creative Commons license, unless indicated otherwise in a credit line to the material. If material is not included in the article's Creative Commons license and your intended use is not permitted by statutory regulation or exceeds the permitted use, you will need to obtain permission directly from the copyright holder. To view a copy of this license, visit http://creativecommons. org/licenses/by/4.0/.

(c) The Author(s) 2022 